\title{
An Examination of the START NOW Dialectical Behavior Therapy- Based Intervention Plus a Behavioral Level System on Male Inmate Misbehavior, Aggressive Behavior, and Mental Health
}

Victoria DiSciullo

West Virginia University, vad0003@mix.wvu.edu

Follow this and additional works at: https://researchrepository.wvu.edu/etd

Part of the Clinical Psychology Commons

\section{Recommended Citation}

DiSciullo, Victoria, "An Examination of the START NOW Dialectical Behavior Therapy-Based Intervention Plus a Behavioral Level System on Male Inmate Misbehavior, Aggressive Behavior, and Mental Health" (2021). Graduate Theses, Dissertations, and Problem Reports. 8147.

https://researchrepository.wvu.edu/etd/8147

This Dissertation is protected by copyright and/or related rights. It has been brought to you by the The Research Repository @ WVU with permission from the rights-holder(s). You are free to use this Dissertation in any way that is permitted by the copyright and related rights legislation that applies to your use. For other uses you must obtain permission from the rights-holder(s) directly, unless additional rights are indicated by a Creative Commons license in the record and/ or on the work itself. This Dissertation has been accepted for inclusion in WVU Graduate Theses, Dissertations, and Problem Reports collection by an authorized administrator of The Research Repository @ WVU.

For more information, please contact researchrepository@mail.wvu.edu. 
An Examination of the START NOW Dialectical Behavior Therapy-Based Intervention Plus a Behavioral Level System on Male Inmate Misbehavior, Aggressive Behavior, and Mental Health

Victoria A. DiSciullo, M.S.

Dissertation submitted

to the Eberly College of Arts and Sciences

at West Virginia University

in partial fulfillment of the requirements for the degree of

Doctor of Philosophy

in Psychology

Elisa Krackow, Ph.D., Chair

Amy Gentzler, Ph.D.

Cheryl McNeil, Ph.D.

Karen Weiss, Ph.D.

Merideth Smith, Ph.D.

Department of Psychology

Morgantown, West Virginia

2021

Keywords: incarceration, inmates, aggressive behavior, mental health, psychological intervention

Copyright 2021 Victoria A. DiSciullo 


\begin{abstract}
An Examination of the START NOW Dialectical Behavior Therapy-Based Intervention Plus a Behavioral Level System on Male Inmate Misbehavior, Aggressive Behavior, and Mental Health
\end{abstract}

\title{
Victoria A. DiSciullo
}

The purpose of this study was to evaluate the impact of the START NOW program + behavioral level system (BLS) in a self-contained therapeutic community (SCTC) on inmate misbehavior at a correctional facility in a southern state. The SCTC includes the START NOW manualized mental health treatment, recreation groups, process groups as needed, and additional positive reinforcement to target inmate prosocial behavior (i.e., level system to obtain privileges). Inmate data was evaluated at 3-months pre-START NOW intervention and 3-months post-START NOW intervention to determine if there were differences in the number of referrals to mental health services for misbehavior, write-ups in the Division of Corrections and Rehabilitation (DCR), referrals to the restrictive housing unit, placement on precaution status, and instances of spontaneous use of force compared with a control group of inmates who were placed in restrictive housing during the same time frame. A series of ANCOVA and chi-square analyses was used to examine any potential differences in these two groups on variables at preintervention versus post-intervention. In contrast to previous studies (Cislo \& Trestman, 2016; Kersten, Cislo, Lynch, Shea, \& Trestman 2015; Shelton \& Wakai, 2011), the current study showed largely nonsignificant results. Although the current study did not find that the START NOW plus behavioral level system group was significantly different from the control group on outcome variables, the intervention group did improve overall. Therefore, further research is necessary to determine the impact of this intervention on inmate disruptive, aggressive, and violent behavior.

Keywords: incarceration, inmates, aggressive behavior, mental health, psychological intervention 


\section{Table of Contents}

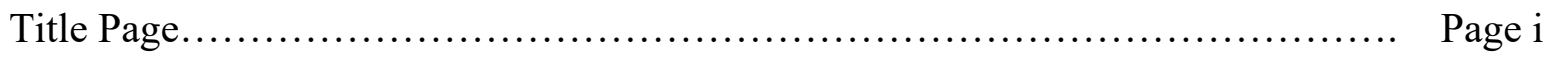

Abstract........................................................... Page ii

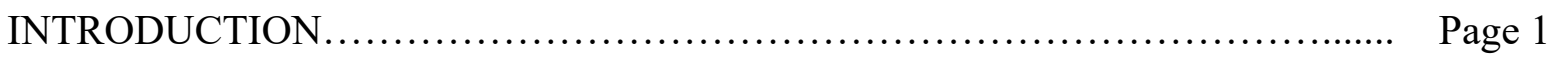

METHODS........................................................ Page 23

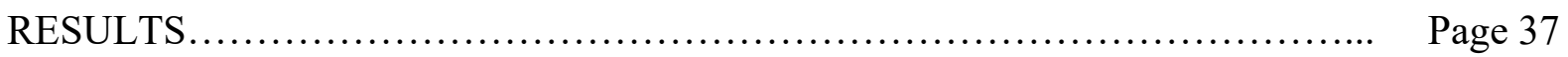

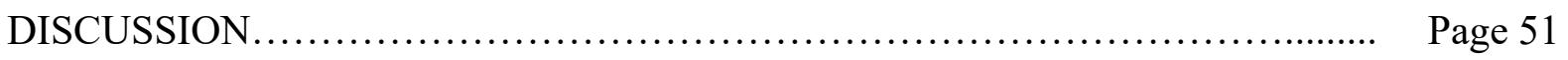

References........................................................ Page 61

Tables............................................................ Page 70 


\section{An Examination of the START NOW Dialectical Behavior Therapy-Based Intervention \\ Plus a Behavioral Level System on Male Inmate Misbehavior, Aggressive Behavior, and \\ Mental Health}

More people are incarcerated in the United States than any other country in the world (Travis, Western, \& Redburn, 2014). In 2016, over two million people in the United States were incarcerated (Kaeble \& Cowhig, 2018). As of 2016, West Virginia had over 11 thousand inmates with approximately 392 per 100 thousand West Virginia residents in prison and 270 per 100 thousand in jail (The Sentencing Project, 2017). West Virginia is ranked at the $22^{\text {nd }}$ highest incarceration rate in the United States (Bronson \& Berzofsky, 2017).

The mental health of inmates incarcerated has been a concern over time. Mental illness has been diagnosed in $37 \%$ of inmates in prisons and $44 \%$ of inmates in jails (Bronson \& Berzofsky, 2017). Further, approximately $14 \%$ of inmates in federal prisons and $26 \%$ of inmates in jails meet criteria for symptoms of psychological distress (i.e., feeling depressed, worthless, hopeless, nervous, restless/fidgety, and feeling that completion of tasks require excessive effort) in a 30-day period (Bronson \& Berzofsky, 2017). The percentage of inmates in prison who have experienced serious psychological distress is three times higher than that of non-incarcerated adults in the United States (Bronson \& Berzofsky, 2017).

A potential indicator of psychological distress or mental illness may be aggressive or self-injurious behavior, which can lead to safety and security concerns in a correctional facility. A study of inpatients in a mental health hospital, who were typically male, life-sentenced inmates, found that most patients who engaged in self-injurious behavior also exhibited aggression (e.g., property damage, verbal or physical aggression; Daffern \& Howells, 2009). These patients typically exhibited aggressive behavior prior to engaging in self-injurious 
behavior (Daffern \& Howells, 2009). A systematic review examining the evidence of cooccurring aggression and self-injurious behavior found that $62 \%$ of the studies included in the review reported heightened aggression in patients who engaged in self-injurious behavior, compared with a control group (O’Donnell, House, \& Waterman, 2015). Additionally, 52\% of the studies reviewed found a positive association between aggression and self-injurious behavior. Finally, the risk of one of these behaviors (i.e., aggression or self-injury) led to an increase in the occurrence of the other behavior (O’Donnell, House, \& Waterman, 2015). Therefore, it appears likely that patients who exhibit one of these behaviors (i.e., aggression or self-injury) will also exhibit the other.

For the large percentage of inmates who have been diagnosed with a mental health disorder or have experienced serious psychological distress, reducing the occurrence of acting out or violent behavior via the use of positive behavioral strategies and acquiring additional adaptive coping skills may be important given that previous research shows that these externalizing behaviors cannot be reduced purely through a punishment strategy (Gendreau, 1996). Although correctional officers have a multitude of responsibilities in the correctional system, they are tasked with maintaining the security and safety of inmates and staff. When safety concerns arise in the correctional system, correctional officers generally choose to use strategies related to punishment, rather than rehabilitative strategies (Gordon, 1999). One punishment strategy correctional officers may choose to use when safety concerns arise is restrictive housing.

\section{Restrictive Housing}

Restrictive housing (also referred to as solitary confinement or segregation) is defined as placing an inmate in a cell alone for between 22 and 24 hours per day with limited social 
interaction, which may last up to years (ACLU, 2014). The term restrictive housing will be used in this document for consistency. An inmate in restrictive housing is usually limited to a toilet, sink, and bunk in a cell with a solid steel door, and is placed in restraints when engaging in social interaction (Lanes, 2009), whereas inmates not placed in restrictive housing may share a cell with one or more inmates, have access to entertainment (e.g., books, coloring) as well as the frequent use of a phone, and spend time in a day room (e.g., watch TV with other inmates on the same unit). Restrictive housing usually includes restriction of privileges (e.g., reading, television, radios), removal of group activities with other inmates (e.g., group meals; ACLU, 2014), and restricted visits from family members (Lanes, 2009). Estimates of the prevalence of restrictive housing in US adult prisons in 2012 indicated that in the 12 months prior, approximately $20 \%$ of inmates in prisons and $18 \%$ of inmates in jails had been placed in restrictive housing (Beck, 2015). From 2008 through 2013, the number of inmates in restrictive housing increased 17\%, while the total rate of incarceration only increased 6\% (United States Government Accountability Office, 2013), which suggests that use of restrictive housing is increasing as a punishment strategy.

Additional security and staff, beyond those employed to work with the general population, are required for each inmate placed in restrictive housing. Therefore, restrictive housing is a costly method of dealing with inmate misbehavior (Browne, Hastings, Kall, \& diZerega, 2015). In addition to the increase in spending associated with restrictive housing, the success of restrictive housing has been underwhelming. Inmates who were placed in restrictive housing for a short period of time due to violent behavior did not show a reduction in violent behavior in the 6 months following restrictive housing (Morris, 2016). Supermax facilities, facilities that use restrictive housing exclusively, do not result in a reduction in inmate-on-inmate 
violence and show mixed results in reducing inmate-on-staff violence (Briggs, Sundt, \& Castellano, 2003). Overall, research finds little to no reduction of violent behavior or misbehavior through the use of restrictive housing, but is still being used regularly in the correctional system.

Although inmates are often placed in restrictive housing as a punishment due to violent behavior, inmates in restrictive housing actually experience more irresistible impulses, as well as feelings related to anger, irritability, resentment, and the possibility of engaging in aggressive behavior more often than inmates in the general prison population (Miller \& Young, 1997). Following restrictive housing, inmates are commonly placed back in the general population with access to other inmates and staff. Therefore, these negative feelings (e.g., anger, irritability) may be an additional concern as they may increase the chance of an inmate engaging in assaultive behavior.

While in the restrictive housing unit, inmates endorsed complaints regarding how they were treated by correctional officers (e.g., use of tear gas, use of force, and degradation by officers; Suedfeld, Ramirez, Deaton, \& Baker-Brown, 1982). With regard to their mental health, inmates experienced a multitude of symptoms during their course of restrictive housing. These symptoms included more depressive symptomology, more issues with psychosocial adjustment (i.e., psychiatric, social, and depressive symptomology), and more anxiety than inmates who were in the general prison population (Zinger, Wichmann, \& Andrews, 2001), and these symptoms appeared to increase as length of time in restrictive housing increased (Suedfeld, Ramirez, Deaton, \& Baker-Brown, 1982).

Inmates in restrictive housing are 3.2 times more likely to engage in self-harm than those who have not been in restrictive housing, and the correlation between self-harm and restrictive 
housing remained significant even after controlling for serious mental illness (Kaba et al., 2014). In $2004,73 \%$ of all suicides in California prisons took place in restrictive housing (American Civil Liberties Union, 2014). Inmates who have been placed in a Clinical Alternative to Punitive Segregation Unit have shown a reduction in self-harm behavior as a result of participation in treatment and increased cooperation between inmates, security, and health staff, in comparison to those inmates who have only been placed in a restrictive housing unit (Glowa-Kollisch et al., 2016).

In summary, the use of restrictive housing is unsuccessful in reducing violent behavior or misbehavior related to inmate-on-inmate or inmate-on-staff conflict, and appears to be related to increased mental health concerns. The implementation of alternative program options such as empirical treatments, might increase positive and prosocial behavior, and have the potential to reduce the misbehavior that leads inmates to restrictive housing, which will also reduce the potential for increased mental health concerns.

\section{Mental Health Treatment in a Correctional System}

This paper will initially discuss the original development, use, and success of DialecticalBehavior Therapy (DBT) with people diagnosed with borderline personality disorder. A discussion regarding the various populations that DBT has since shown effectiveness with, including correctional populations will follow. Next, a review of the consistency between the DBT model and the Risk-Need-Responsivity (RNR) model will be presented. Finally, a review of various programs, including the START NOW intervention, which originated from DBT will be discussed.

\section{Dialectical-Behavior Therapy (DBT)}


DBT is a mental health treatment that originated from cognitive-behavioral therapy with eastern cultural influences, specifically for those diagnosed with borderline personality disorder who were suicidal (Linehan, 2015), but was later adapted for other populations, including the correctional population (Eccleston \& Sorbello, 2002; Sampl, Wakai, \& Trestman, 2010; Shelton, Kesten, Zhang, \& Trestman, 2011; Shelton, Sampl, Kesten, Zhang, \& Trestman, 2009). DBT focuses on emotion dysregulation and works to improve interpersonal, behavioral, thought, and emotional patterns that affect daily life. DBT is based on a biosocial theory, which theorizes that emotion dysregulation is impacted by both biology (e.g., heredity, early learning, brain development) and the person-environment interaction (e.g., invalidating environment, lack of appropriate interpersonal models). In order to improve emotion dysregulation, DBT involves teaching clients various skills (i.e., mindfulness skills, interpersonal effectiveness skills, emotional regulation skills, and distress tolerance skills). Mindfulness skills are taught to engage the client to focus on the present and teaches them to accept each moment as is comes, without judgement (Linehan, 2015). Interpersonal effectiveness skills are taught to help the client develop strategies to say "no" and ask for needs, even in situations that are difficult or uncomfortable for them. Emotion regulation skills are comprised of the ability to understand, name, and identify emotions in order to address the dysfunctional behaviors that many clients exhibit as a result of emotion dysregulation. Finally, distress tolerance skills are taught to teach the client how to accept and tolerate negative emotions using crisis survival skills (e.g., distraction, meditation using present body sensations, choosing to accept reality; Linehan, 2015).

As patients develop these skills, they learn to be present in the moment, improve interpersonal relationships, recognize and label emotions, and limit maladaptive behaviors (e.g., impulsive and suicidal behaviors; Linehan, 2015). DBT encompasses simultaneous group and 
individual skills training, individual therapy (also termed case management), and consultation as necessary (Linehan, 2015). In Linehan's standard adult DBT protocol, skills are learned over a period of 24 weeks, and treatment modules can be repeated such that the intervention lasts up to 1 year (Linehan, 2015). In a variety of randomized controlled trials, DBT has been found to decrease suicidal behavior, including number of suicide attempts, non-suicidal self-harm behavior, substance use, hopelessness, depression, anxiety, and use of psychotropic medication (Linehan, Armstrong, Suarez, Allmon, \& Heard, 1991; Linehan, Tutek, Heard, \& Armstrong, 1994; van den Bosch, Verheul, Schippers, \& van den Brink, 2002; Verheul, van den Bosch, Koeter, de Ridder, Seijnen, \& van den Brink, 2003; Koons, Chapman, Betts, O’Rourke, Morse, \& Robins, 2006; Pistorello, Fruzzetti, MacLane, Gallop, \& Iverson, 2012).

Linehan, Armstrong, Suarez, Allmon, and Heard (1991) conducted a study in which they recruited 44 participants between 18- and 45-years-old, all of whom met criteria for borderline personality disorder and had two or more occurrences of self-harm within a 5-year period. Participants were matched on several variables (i.e., self-harm occurrences, age, prognosis, and hospitalizations) and half were assigned to the DBT treatment condition, while the other half were assigned to the treatment as usual control condition. The DBT condition consisted of weekly group therapy ( $2 \frac{1}{2}$ hours) and weekly individual therapy ( 1 hour). Group therapy included training in interpersonal skills, emotion regulation skills, and distress tolerance skills. Individual therapy included exposure to emotional cues, contingency management, behavioral skills training, cognitive restructuring, reflection, empathy, and acceptance. Participants also had telephone contact between sessions with their individual therapist. All therapists in the DBT condition were supervised by Dr. Linehan, who conducted fidelity checks (i.e., listening to audio recordings of sessions) and supervised the treatment weekly. Treatment as usual (TAU) 
consisted of researchers providing participants with a list of mental health referrals from which to choose. Individual psychotherapy was attended by $73 \%$ of the participants in the TAU condition. It is unknown to this reader what therapy components were included in the TAU psychotherapy. All participants were assessed on a variety of measures (i.e., self-harm occurrences, treatment contact, suicidal ideation, depression, hopelessness, and reasons for living) at pretreatment, 4 months into treatment, 8 months into treatment, and 12 months after the start of treatment. Participants who received DBT exhibited fewer self-harm occurrences, spent less time in a psychiatric hospital, and were less likely to drop out of therapy than TAU participants (Linehan, Armstrong, Suarez, Allmon, \& Heard, 1991).

Due to the effectiveness of DBT with women with borderline personality disorder who have a history of self-harm, DBT has been employed with other populations including psychiatric, corrections, and adolescent populations. In psychiatric populations (women with borderline personality disorder who have a criminal history, but were not incarcerated), participation in DBT has led to reductions in self-harm, irritability, hopelessness, impulsivity, suicidal ideation, and improvement in functioning, as well as the ability to transfer to a less secure facility following completion of one year of DBT (Low, Jones, Duggan, MacLeod, \& Power, 2001). However, these effects often diminished when the environment changed (e.g., following a move to a different facility) and additional therapy was necessary (Low, Jones, Duggan, MacLeod, \& Power, 2001). Furthermore, DBT has exhibited effectiveness with a male borderline personality disorder population displaying antisocial behavior, such that self-harm, aggression, rule-breaking, and criminal offending were all reduced following 12 months of DBT treatment (Wetterborg et al., 2020). 
DBT has also demonstrated effectiveness with an adolescent sample (46\% male; McCredie, Quinn, \& Covington, 2017). Although this sample may be different in age, many outcome variables in this study are similar to those problematic behaviors that would be relevant to a correctional setting (e.g., rule-breaking behavior). Following completion of the DBTAdolescent (DBT-A) program, none of the adolescents in a residential treatment setting scored in the clinically significant range on the Youth Self-Report, and scores on many scales decreased (e.g., total problems, rule-breaking behavior, thought problems, attention problems) from pretreatment to post-treatment (McCredie, Quinn, \& Covington, 2017). Additionally, there was a reduction in the number of mental health diagnoses for which the adolescents met criteria, as well as a reduction in symptoms for current diagnoses (e.g., substance abuse, major depressive disorder, eating disorders). Adolescents most commonly endorsed daily use of distress tolerance and emotion regulation skills, which were the two DBT skills that both showed the greatest effects (McCredie, Quinn, \& Covington, 2017). This finding appears particularly important, as these adolescents endorsed responses which demonstrated that these skills were helpful to them outside of the group skills trainings.

DBT has been adapted for use with correctional populations with success (Eccleston \& Sorbello, 2002; Shelton, Kesten, Zhang, \& Trestman, 2011; Shelton, Sampl, Kesten, Zhang, \& Trestman, 2009) and is consistent with the Risk-Need-Responsivity (RNR) model, although many adaptations are necessary due to the limitations of a correctional setting (e.g., fifth-grade reading level, coping behaviors and examples applicable to an offender population; Sampl, Wakai, \& Trestman, 2010). Offender treatment which follows the RNR model, demonstrates effectiveness in reducing overall recidivism rates for offenders (Bonta \& Andrews, 2007). The RNR model was conceptualized as a basis for the construction of empirically supported 
treatment of offenders. The RNR model provides a set of principles (risk, need, and responsivity) that are used to guide the process of offender rehabilitation regardless of the specific rehabilitation methods, taking into account the limitations of a correctional setting (Bonta \& Andrews, 2007). The principle of "risk" identifies who should be treated, such that more services should be provided to offenders who have a higher risk of recidivism (Bonta \& Andrews, 2007). The intensity and frequency with which DBT was used in studies housed in a correctional setting shows evidence that DBT may be especially impactful for higher-risk offenders, aligning with the "risk" principle (Tomlinson, 2018). The second principle, "need," identifies the importance of criminogenic needs being recognized and targeted throughout treatment (Bonta \& Andrews, 2007). Various criminogenic needs are identified in the use of DBT in correctional settings, such as restructuring thinking which supports criminal behavior (e.g., "I had to steal; I had no choice”), addressing procriminal friendships (i.e., surrounding oneself with others who engage in criminal behavior), and improving self-control and sensation seeking behavior (Tomlinson, 2018). The third and final principle of the RNR model, "responsivity," identifies the most impactful treatment options for this particular population (e.g., cognitive-behavioral treatments that are idiographic and tailored to a specific offender's abilities; Bonta \& Andrews, 2007). DBT is flexible enough to meet the needs of offenders, aligning with the "responsivity" principle (Tomlinson, 2018). What follows is a review of DBT studies that have been implemented in a correctional setting.

DBT has been modified for use in a correctional setting with the purpose of reducing aggressive and violent behavior in the prison system. The Real Understanding of Self-Help (RUSH) program was adapted from the original DBT treatment manual and specified for use in a male prison setting (Eccleston \& Sorbello, 2002). The RUSH program includes the original four 
DBT modules (e.g., Mindfulness, Distress Tolerance, Emotion Regulation, and Interpersonal Effectiveness), with simplified titles. Modifications to the original DBT manual, include simplified language, a different order of modules, simplified handouts and acronyms, creation of different examples (to apply to the offender population), added activities, and includes group therapy only. Group therapy skills training occurred two times per week for a total of 10 weeks and individual therapy that did not align with DBT principles (i.e., concerns inappropriate for the group) was offered, as needed. The goal of this program is to give inmates with either a previous borderline personality disorder diagnosis or with skills deficits consistent with borderline personality disorder, the opportunity to learn more adaptive skills, and to reduce self-harm and suicide. Results from pre-treatment to post-treatment (following the last therapy session, week 10) were nonsignificant with regard to depressive, anxiety, and stress symptoms. Offenders did however, note motivation to use the skills learned and commitment to treatment (Eccleston \& Sorbello, 2002).

A second DBT modification, DBT-Corrections Modified (DBT-CM), had positive effects in the correctional system (Shelton, Sampl, Kesten, Zhang, \& Trestman, 2009; Shelton, Kesten, Zhang, \& Trestman, 2011). This treatment outcome investigation included inmates who had exhibited impulsive behavior problems, such that the inmates had received many write-ups for problematic behavior, and staff indicated difficulty managing these inmates prior to treatment. The DBT-CM treatment included group skills training for a total of 16 weeks plus individual case management or individual DBT-CM coaching once per week for 30 minutes. Case management and individual skills adjuncts were not defined by the researchers; therefore, it is unclear how they may have differed from each other. After offenders $(72.5 \%$ male $)$ completed DBT-CM (indicated as the completion of $50 \%$ or more of the treatment), they exhibited a 
reduction in disciplinary tickets (Shelton, Sampl, Kesten, Zhang, \& Trestman, 2009). This applied whether the offender was in the individual case management condition or the DBT-CM coaching condition. They also exhibited decreased aggression, increased adaptive coping skills, and improved affect at follow up (Shelton, Sampl, Kesten, Zhang, \& Trestman, 2009). Male incarcerated adolescents who participated in DBT-CM exhibited reduced physical aggression, reduced disciplinary tickets (a disciplinary action for behavior that has violated Division of Corrections and Rehabilitation (DCR) policies), and increased use of distancing as a coping strategy at post-test (i.e., distancing themselves from a short-term stressful situation; Shelton, Kesten, Zhang, \& Trestman, 2011).

Throughout the development of DBT-Corrections Modified (DBT-CM), various adjustments to the original DBT manual were necessary for the treatment to be implemented in this particular setting (Sampl, Wakai, \& Trestman, 2010). DBT-CM consisted of 32 group therapy sessions twice weekly, lasting approximately one hour. Individual therapy sessions took place once weekly, for approximately 30 minutes, and participants were randomized to individual therapy that either focused on developing DBT skills or case management issues (e.g., medical issues, employment). Additionally, the manual language was re-written to ensure a fifthgrade reading level and examples were changed to ensure that they were applicable to an offender population. Examples of coping behaviors were edited, as opportunities for use in a correctional setting are limited (e.g., buying flowers and lighting candles was changed to looking at pictures in a magazine and pictures of family members). Extensive collaboration with security staff was necessary to ensure that they understood the purpose of the treatment and to address any resistance from staff. Therapist adherence to the treatment was monitored through direct 
observation once per month, as videotaping is not an option in a correctional setting (Sampl, Wakai, \& Trestman, 2010).

Despite positive effects of the DBT-CM approach, extensive adjustments to the original DBT manual were deemed necessary by other researchers to implement a DBT-based treatment in a correctional setting such that ultimately a new treatment manual was developed and named START NOW (Sampl, Wakai, \& Trestman, 2010). Due to limited resources and staff, safety limitations, inmate abilities, and necessary flexibility needed in the correctional setting (e.g., lock down situations, inmates transferred to other facilities), this new treatment manual was developed for easier implementation in correctional settings (Sampl, Wakai, \& Trestman, 2010). START NOW Intervention

START NOW has been developed through adaptations and modifications of DBT, specifically for a correctional setting with limited resources. This program does not utilize Linehan's entire DBT model, but instead, includes various skills developed through DBT. START NOW includes 32 group therapy sessions to be distributed twice weekly (Sampl, Wakai, Trestman, \& Keeney, 2008). The program includes four modules: interpersonal skills, understanding/coping with emotions, problem-solving, and future-oriented skills (Sampl, Wakai, Trestman, \& Keeney, 2008).

The START NOW program is comprised of four modules, and the skills included in these modules have been evaluated with a variety of populations (Clark, 2015; Dixon, Heppner, Petersen, \& Ronning, 1979; Frey \& Weller, 2000; Garofalo, Velotti, \& Zavattini, 2018; Hayakawa, 2009; Hesser et al., 2017; Mennin, Fresco, O’Toole, \& Heimberg, 2018; Tartaro, 2015). Interpersonal skills and assertiveness training have resulted in improvements in aggressive behavior and prosocial behavior (Frey \& Weller, 2000), as well as a reduction in self- 
harm behavior (Hayakawa, 2009). Emotion regulation has been connected to aggression in populations of male inmates and community dwelling males, such that emotion dysregulation helped to explain increased anger, aggression, and hostility (Garofalo, Velotti, \& Zavattini, 2018). Treatments that include an emotion regulation component have been able to reduce psychological and physical interpersonal violence, depression, anxiety, and aggression (Hesser et al., 2017). A randomized controlled trial of emotion regulation therapy demonstrated improvements in symptoms of generalized anxiety disorder and major depressive disorder in participants, with effects maintained through nine-month follow up (Mennin, Fresco, O'Toole, \& Heimberg, 2018). Problem-solving skills training has led to an increase in participants' abilities to create alternative solutions to common problems, more detailed solutions to problems, and participants reported that they perceived their problem-solving abilities as less impulsive following training (Dixon, Heppner, Petersen, \& Ronning, 1979). Inmates involved in transition or re-entry planning, which may include planning for jobs, mental health treatment, housing, and/or future-oriented goal setting, spent more time in the community before re-offending (Tartaro, 2015) and exhibited a reduced risk of reconviction (Clark, 2015).

In addition to the four major components of the START NOW program, START NOW teaches inmates to employ a functional assessment of their behavior ( $\mathrm{ABC}$ system) to evaluate a variety of situations (Sampl, Wakai, Trestman, \& Keeney, 2008). A functional assessment is generally used to determine how certain variables may impact a specific behavior (Welches \& Pica, 2005). In an "ABC" system of functional assessment, the "B" refers to the behavior, the "A" refers to the antecedent or the circumstances that precede the behavior, and the " $\mathrm{C}$ " refers to the consequence or what follows (and may reinforce) the behavior (Welches \& Pica, 2005). This allows the patient or practitioner to determine under what conditions a behavior occurs, and what 
a person may gain from engagement in a particular behavior. Welches and Pica (2005) observed that patient inclusion in a functional assessment of behavior, as opposed to a functional assessment of behavior being conducted by an outside observer such as a clinician, improved the accuracy of the assessment and validated patients' feelings regarding their symptomology (see also Kurtz et al., 2003 and Shayne \& Miltenberger, 2013 for additional effectiveness with foster and biological parents).

The main goal of START NOW is positive behavior change for inmates through the development of various skills such as decision making and judgement, new coping strategies, emotion regulation skills, improved self-control, and recognition of emotion and social cues (Shelton \& Wakai, 2011). To target improved self-control, inmates are taught mindfulness skills (e.g., being present, using senses) and how to use functional assessment to determine the antecedents and consequences of their behavior (Sampl, Trestman, \& Krauss, 2013). Emotion regulation is targeted through the ability to recognize emotions, verbalize emotions, and understand the influence of an individual's perspective of a situation on emotions (Sampl, Trestman, \& Krauss, 2013). Interpersonal and communication skills are targeted through acceptance of feedback, assertiveness training, ability to set boundaries, and requests for support (Sampl, Trestman, \& Krauss, 2013). To focus on an inmate's future, START NOW includes setting and breaking down goals, problem-solving barriers, and instilling hope for the future (e.g., finding a job, finding housing; Sampl, Trestman, \& Krauss, 2013).

START NOW is structured to include 32 group sessions over the course of four months (two per week for 75 minutes; Shelton \& Wakai, 2011). New participants may begin the program at the start of any particular program unit; therefore, the four sections may be in a different order for some participants (Sampl, Trestman, \& Krauss, 2013). START NOW facilitators are 
expected to approach the group skills trainings with Motivational Interviewing (MI) strategies such as, eliciting change talk, working through ambivalence, using double-sided reflections, and summarizing (Sampl, Trestman, \& Krauss, 2013). The purpose of using MI strategies is to elicit behavior change, which can lead to a reduction in ambivalence (Rollnick \& Miller, 1995). Although some MI strategies are used in the START NOW program, the program is not identified as a MI therapeutic intervention and has not been evaluated as such. Fidelity checks have been built into the program to ensure treatment adherence (Shelton \& Wakai, 2011). These procedures include monthly observations of one START NOW session by a START NOW trainer, who rates each START NOW facilitator on quality assurance. If needed, additional training is provided to facilitators following these fidelity checks (Shelton \& Wakai, 2011).

\section{START NOW Outcome Studies}

In the first START NOW treatment outcome study, Shelton and Wakai (2011) examined initial outcome data for 26 inmates who completed the intervention. The purpose of this study was to use this information to further advance the START NOW program and to obtain information which could be used to make recommendations to facilities regarding program improvements. Consistent with other START NOW studies, the START NOW program included 32 group sessions over the course of four months (two per week for 75 minutes). START NOW generally improved inmate behavior and mental health, such that following completion of the START NOW program, there was a decrease in the number of disciplinary tickets (a disciplinary action for behavior that has violated DCR policies), number of days in the restrictive housing unit, and number of admissions to the mental health unit compared to three months prior to treatment (Shelton \& Wakai, 2011). Prescribed psychotropic medications increased following the START NOW intervention; however, greater participation in START NOW sessions resulted in 
those prescriptions being deemed no longer necessary, such that they were eventually terminated (Shelton \& Wakai, 2011). In this study, improvement was determined by a reduction in pre-post group means, as opposed to statistical analyses (Shelton \& Wakai, 2011).

In a recent study of the START NOW program, researchers analyzed outcome data from participants who were involved in this program over a period of four years (Kersten, Cislo, Lynch, Shea, \& Trestman, 2015). Participants were inmates in seven correctional facilities who were at least 18 years old and incarcerated for one month or more following involvement in the START NOW program. Inmates were given the opportunity to participate in the program or portions of the program multiple times; therefore, the study included data from 100 people who participated more than once. Only post-program data were collected and the amount of time following treatment completion varied from 30 - 180 days, with a mean of 165 days. The dependent variable was number of disciplinary reports, and the main independent variable was number of START NOW sessions completed. Authors also examined the effect of additional variables that may have impacted these variables (i.e., inmate security risk and psychiatric diagnoses). As the number of sessions attended increased, disciplinary reports obtained in the six months following the program decreased, and those inmates who were deemed a higher security risk within the facility experienced the greatest decrease (Kersten, Cislo, Lynch, Shea, \& Trestman 2015). Further, an increase in sessions attended by inmates was associated with a reduction in the number inpatient psychiatric days post-treatment (Cislo \& Trestman, 2016). This relation applied until approximately 17 sessions, when each additional session was no longer associated with an additional reduction (Cislo \& Trestman, 2016).

In addition to the positive quantitative impact of START NOW, qualitative research has indicated that inmates reported satisfaction with the START NOW program and stated that they 
would recommend this program to others (Shelton \& Wakai, 2011). This is important because inmates are generally not mandated to mental health treatment for behavior difficulties while incarcerated; therefore, inmate motivation and satisfaction to participate is an integral part of a successful intervention in a correctional setting. A few inmates reported dissatisfaction due to the quick pace of the program, leading to difficulty effectively learning the material (Shelton \& Wakai, 2011). Facilitator consistency was associated with higher program satisfaction (Shelton \& Wakai, 2011).

In summary, START NOW is adapted from DBT, an evidence-based treatment, which has shown effectiveness in a variety of populations (Eccleston \& Sorbello, 2002; Low, Jones, Duggan, MacLeod, \& Power, 2001; McCredie, Quinn, \& Covington, 2017; Shelton, Kesten, Zhang, \& Trestman, 2011; Shelton, Sampl, Kesten, Zhang, \& Trestman, 2009). Previous studies have evaluated the impact of the START NOW program on the number of disciplinary tickets, number of days in the segregation unit, number of admissions to the mental health unit, number of and adherence to psychotropic medication (Shelton \& Wakai, 2011), inmate security risk, and number of psychiatric diagnoses (Kersten, Cislo, Lynch, Shea, \& Trestman, 2015). Thus, implementing the use of START NOW may lead to positive mental health outcomes that are generally seen with the use of DBT in various populations, and may be useful as a rehabilitative strategy, ultimately reducing inmate recidivism in the long term. The program is behaviorally based, and programs that have been shown to reduce inmate recidivism have typically included behavioral-based skills and training on the use of positive reinforcers (Gendreau, 1996).

In addition to the use of the START NOW intervention, one particular correctional facility has added a behavior reward system. Token economies and other behavior reward systems have previously been used with a variety of populations, outside of a forensic setting, 
and have been found to be effective (Burdon, St. De Lore, Dang, Warda, \& Prendergast, 2013; Glowacki, Warner, \& White, 2016; Pritchard, Penney, \& Mace, 2017). For example, inmates in a substance abuse treatment program were provided with a token economy, such that they were given points to save and exchange contingent on engagement in prosocial behaviors and avoidance of antisocial behavior (e.g., no disciplinary issues, achievement of treatment goals, work attendance) and exhibition of commitment to treatment (e.g., attendance in group sessions, completion of homework assignments; Burdon, St. De Lore, \& Prendergast, 2011). Depressive symptoms decreased more for female participants involved in the token economy than female participants in the treatment only condition, and criminal thought patterns decreased more for the male participants involved in the token economy than male participants in the treatment only condition (Burdon, St. De Lore, Dang, Warda, \& Prendergast, 2013). Although there have been anecdotal reports regarding the implementation of diversionary treatment units in correctional settings, published research concerning the impact of these units was not located during literature searches.

Further, reward systems have been implemented extensively with positive effects in inpatient psychiatric settings and with children with extensive behavior problems (Atthowe \& Krasner, 1968; Van Allen, 1970; Broden, Hall, Dunlap, \& Clark, 1970). Recently, Pritchard, Penney, and Mace (2017) developed a reward system for students with severe problem behavior contingent on academic work (e.g., completion, cooperation, on task behavior) per 30-minute periods, that allowed access to a reward menu (i.e., list of rewards for a student to choose from) developed based on students' preferences. They also developed a level system, in which moving up the levels was contingent on increased academic reward points earned, increased attention to school tasks, absence of problematic sexual behavior, and absence of problematic behavioral 
occurrences. As participants moved up levels, they gained access to new activities, community outings, and were able to exchange their points for money. Implementation of a combination of the token economy and level systems (e.g., exhibition of prosocial behaviors to gain more privileges) exhibited positive effects, such that as students reached higher levels in the program, there was a decrease in problem behavior (Pritchard, Penney, \& Mace, 2017).

In addition to the positive impact of a token economy on inmate and student samples, positive effects have also been found with multiple inpatient psychiatric samples (Glowacki, Warner, \& White, 2016). A review of studies of inpatient psychiatric facilities that used a token economy to decrease negative behaviors (e.g., aggression, drug use, violence) or negative symptoms (e.g., limited social interaction, flat affect, limited pleasure in activities) indicated that each study in the review found that token economies decreased negative behavior (Glowacki, Warner, \& White, 2016).

Although multiple studies have evaluated START NOW, the weaknesses and limitations of these designs suggest the need for additional research regarding the START NOW program. First, one of the available articles on the START NOW program focuses on providing information regarding the effective implementation of the program, rather than a statistical evaluation (Sampl, Wakai, Trestman, \& Keeney, 2008). Of the available outcome studies, only one study analyzed data by inmate (Shelton \& Wakai, 2011), and this study researched the outcome variables using a descriptive rather than statistical approach. The sample size of the single study that analyzed data by inmate was small $(N=26$; Shelton \& Wakai, 2011), which limits the ability to make claims about outcomes. The other studies used a frequency count of participation events in the program in the analyses (as inmates were able to utilize the program multiple times; Cislo \& Trestman, 2016; Kersten, Cislo, Lynch, Shea, \& Trestman, 2015). This 
use of participation events means that some inmates' data were counted multiple times in analyses. Additionally, only post-treatment data were obtained (no pre-post data) and the timeframe in which the post-treatment data was gathered was inconsistent across participants (Cislo \& Trestman, 2016; Kersten, Cislo, Lynch, Shea, \& Trestman, 2015). Next, these studies have used disciplinary action as an outcome measure, but all inmates, even those without excessive disciplinary action, had the opportunity to be involved in the program (other than those with the highest security classification; Cislo \& Trestman, 2016; Kersten, Cislo, Lynch, Shea, \& Trestman, 2015). Therefore, there is a lack of knowledge regarding how this program works with inmates who exhibit largely violent, aggressive, and disruptive behavior while incarcerated. Finally, all four of the previous studies have been conducted in the state of Connecticut only, which limits the ability to generalize the findings to other areas of the United States.

Additionally, there is a unique opportunity to evaluate the START NOW intervention plus a behavioral level system for added behavior modification in addition to the START NOW program. Overall, it appears that undertaking a study of START NOW with a larger sample size of inmates who exhibit significant violent, aggressive, and disruptive behavior in another state in the US may be advantageous for evaluating the impact of the program.

\section{The Current Study}

The current study evaluated the impact of the START NOW intervention plus behavioral level system (BLS) on inmate disruptive, violent, and aggressive behavior at a correctional facility in a southern state. The self-contained treatment community (SCTC) uses the START NOW intervention for mental health treatment, along with additional positive reinforcement to target inmate prosocial behavior (i.e., level system to obtain privileges). This study analyzed data for all inmates who have participated in the SCTC since its inception (i.e., START NOW + BLS) 
at this facility, to determine the impact of the START NOW intervention plus positive reinforcement for prosocial behavior (level system) on inmate disruptive, violent, and aggressive behavior, as well as inmate mental health prior to and following completion of the program.

Multiple hypotheses were developed based on previous START NOW research (Shelton \& Wakai, 2011; Kersten, Cislo, Lynch, Shea, \& Trestman, 2015). First, a reduction in the number of referrals to mental health services for misbehavior for inmates who complete 17 or more sessions of the START NOW intervention is hypothesized to occur at a higher rate compared with the control group. Shelton and Wakai (2011) found that START NOW participants spent fewer days in the mental health unit following completion of the intervention. These findings are expected to be related to the number of referrals to mental health services for misbehavior. We anticipate similar findings, such that following completion of the START NOW treatment, there will be a reduction in the number of referrals to mental health services for inmate misbehavior. Next, a reduction in the number of write-ups for misbehavior in the DCR for inmates who complete 17 or more sessions of the START NOW treatment is hypothesized to occur at a higher rate compared with the control group. Shelton and Wakai (2011) and Kersten, Cislo, Lynch, Shea, and Trestman (2015) found a reduction in the number of write-ups for misbehavior following completion of the START NOW intervention. Finally, a reduction in the number of referrals to the restrictive housing unit for inmates who complete 17 or more sessions of the START NOW intervention is anticipated to occur at a higher rate compared with the control group. Shelton and Wakai (2011) found that START NOW participants spent fewer days in the restrictive housing unit following completion of the START NOW intervention. We hypothesize that similar findings will occur, such that following completion of the START NOW treatment, there will be a reduction in the number of referrals to the restrictive housing unit. 
Previous research has not yet evaluated the impact of the START NOW intervention on inmates' instances of spontaneous use of force or placement on precaution status (i.e., suicide watch). Therefore, there are no current hypotheses in place regarding the impact of the START NOW intervention on these variables.

\section{Method}

\section{Participants}

This study included data from 88 male offenders, age 18 or older. There were no female offenders included in the current study because this particular correctional facility only houses male inmates. The participants included the total number of male offenders who completed the SCTC including the START NOW intervention from February 2016 through March 2020, which totaled 45 inmates. Completion of the SCTC including the START NOW intervention was defined as inmates who completed 17 sessions or more of the START NOW intervention. Previous START NOW research found that an increase in the number of sessions attended led to a reduction in the number of inpatient psychiatric days post-treatment until approximately 17 sessions, when each additional session was no longer associated with an additional reduction (Cislo \& Trestman, 2016). The inclusion of inmates who completed 17 or more START NOW sessions allowed for a larger sample size to provide adequate power for statistical analyses.

The only inmates whose data were not included in the START NOW + BLS group were those who had completed the START NOW intervention multiple times. Additionally, 43 participants were included as a control group. These inmates were housed at the same correctional facility as the inmates in the SCTC throughout the entire observation period (i.e., from pre-intervention to post-intervention), and were placed in restrictive housing at least once 
during the same pre-intervention period as an inmate in the START NOW + BLS intervention group. The sample size was adequate for required power.

The average age of participants in the START NOW + BLS group was 32.47 years, with an age range of 22-56 years. The identified race/ethnicity of the participants in the START NOW + BLS group was as follows: Caucasian (41 participants), African American/Black (3 participants), and American Indian/Alaska Native (1 participant). No participants identified as Asian/Pacific Islander, Biracial, or Hispanic/Latinx. The education level of participants in the START NOW + BLS group was as follows: completion of elementary school (1 participant), completion of middle school (20 participants), completion of high school (i.e., diploma; 6 participants), and completion of a high school equivalency degree (i.e., GED; 18 participants). In the START NOW + BLS group, approximately $62 \%$ of participants were diagnosed with a personality disorder, $47 \%$ with an impulse control disorder, and $64 \%$ with a substance use disorder. Inmates were incarcerated for an average of 1463.07 days (48.77 months) before entering the SCTC, with a range from 370-4626 days (12.33-154.20 months). There were a variety of offenses among the inmates in the START NOW + BLS group; however, approximately $49 \%$ of inmates had an interpersonal offense (e.g., first degree murder, robbery, sexual assault).

The average age of participants in the control group was 35.72, with an age range of 2158. The identified race/ethnicity of the participants in the control group was as follows: Caucasian (40 participants) and African American/Black (3 participants). No participants identified as Asian/Pacific Islander, American Indian/Alaska Native, Biracial, or Hispanic/Latinx. The education level of participants in the control group was as follows: completion of elementary school (0 participants), completion of middle school (11 participants), 
completion of high school (i.e., diploma; 14 participants), and completion of a high school equivalency degree (i.e., GED; 18 participants). In the control group, approximately $37 \%$ of participants had a personality disorder, $28 \%$ had an impulse control disorder, and $44 \%$ had a substance use disorder. The control group participants were each matched with a START NOW + BLS group participant on their pre-intervention to post-intervention time frame. Thus, inmates in the control group were incarcerated for an average of 2329.70 days (77.66 months) with a range from 206-7392 days (6.87-246.40 months) before their matched participant entered the SCTC. There were a variety of offenses among the inmates in the control group; however, approximately $67 \%$ of inmates had an interpersonal offense (e.g., first degree murder, robbery, sexual assault).

In order to be accepted into the SCTC including the START NOW intervention, inmates contacted a mental health staff member and expressed a willingness to engage in a therapeutic process and requested admittance to the SCTC. Inmates' records were reviewed by a panel of the Division of Corrections and Rehabilitation personnel (classification director, Associate Superintendent of Programs, Associate Superintendent of Security, and mental health staff of the SCTC), who all concurred to admit the inmate to the program. Inmates may have been accepted into the SCTC for many reasons, including a history of multiple write-ups, a history of restrictive housing, or recent non-suicidal self-injury. If an inmate posed a security threat (e.g., inability to reside in the same facility as another inmate due to violence, predatory behavior), the inmate could have been denied admittance to the SCTC by the Associate Superintendent of Security based on these security concerns. Upon admittance, inmates were transported to a specific correctional facility in this state to participate in the SCTC, including the START NOW intervention plus BLS. 


\section{Materials}

START NOW Facilitator and Participant Manuals (Sampl, Trestman, \& Krauss, 2013).

The START NOW Facilitator Manual provides guidance and information for SCTC staff leading group sessions. The manual provides therapeutic content for all of the 32 START NOW sessions, and is designed to teach clients therapeutic skills for all four START NOW modules [i.e., My Foundation: Starting with Me (8 sessions), My Emotions: Dealing with Upset Feelings (10 sessions), My Relationships: Building Positive Relationships (8 sessions), and My Future: Setting and Meeting my Goals (4 sessions)]. The START NOW Participant Manual follows the Facilitator Manual, and provides inmates with handouts for practice exercises and session activities (Sampl, Trestman, \& Krauss, 2013).

START NOW Fidelity Checklist (Sampl, Trestman, \& Krauss, 2013). The START NOW Fidelity Checklists are designed to be completed by the START NOW facilitator who is not leading the group, and are used per session. The purpose of these checklists is to ensure that facilitators are maintaining the integrity of the treatment, and covering all necessary components. The fidelity checklists address both the content of the program (e.g., review of the four START NOW units, discussion of resistance to change) and the process in which the facilitator provides the content (e.g., verbal reinforcement for positive change, attempts to elicit change talk). START NOW Fidelity Checklists were designed to include a variety of ratings that reference completion (i.e., none, some, fully), and also include an additional section that allows for comments, if needed (Sampl, Trestman, \& Krauss, 2013).

Behavioral Level System (Roush, Bryson, \& Weaver, 2012). The SCTC's level system is identical to that used in the WVDCR. This document was designed to provide staff with a guide to the privileges and requirements of a level system in a correctional setting. The privileges 
addressed in the level system are obtained with appropriate participation in the START NOW intervention (e.g., eat in dayroom, get a job, receive multiple visits per month). The behavioral level system is observational, and both mental health staff and correctional officers observe inmate behavior while in the SCTC (Roush, Bryson, \& Weaver, 2012).

\section{Procedure}

General overview. The state in which the facility resides approved the use of their precollected data. Therefore, informed consent to access that state's records was not necessary. Additionally, this research was approved by the West Virginia University Institutional Review Board. The inmates involved in the SCTC live and engage in therapy all on one particular unit in the facility. They are expected to participate in START NOW treatment groups (four times per week for three months; approximately 60 minutes per session), as well as recreational groups (e.g., painting) to engage and practice the skills they are learning with the other inmates in the program. As they participate in this program, inmates are expected to refrain from self-harm and disciplinary infractions, which help them move up in the level system. These procedures occur for each inmate involved in the SCTC, but the principal investigator was not involved in the therapeutic processes (i.e., running groups, or engagement with participants in the therapy).

START NOW Intervention. The START NOW intervention was conducted by two START NOW therapists, one with a Master's degree in counseling (currently under supervision for licensing), and another who is a licensed social worker in the process of obtaining a $\mathrm{PhD}$ in psychology. Therapists led two START NOW group sessions per week, with two additional START NOW question and answer sessions to respond to inmate questions and clarify any confusion about START NOW skills. The group format was open, with inmates entering the SCTC any time there was an open space in the program (6 positions at any one time). Inmates 
attended their first session of the START NOW intervention within one week of entering the SCTC.

The first unit the therapist presented in the START NOW intervention, termed My Foundation: Starting with Me, consists of eight sessions and is concentrated on the development of self-control and increased positive coping skills. Skills that therapists included in this unit were focusing skills (e.g., counting breaths, imagery) and a functional assessment of behavior (i.e., ABC system; Sampl, Trestman, \& Krauss, 2013). In this unit, therapists taught inmates the following skills: exercises to be present in the moment such as use of imagery (e.g., imagining oneself doing current activities), development of self-acceptance, use of positive self-talk (e.g., acceptance of themselves), acceptance of difficult situations (e.g., death of loved one), and identification of personal values. In this unit, both development of self-care skills and exploration of spirituality are included in the START NOW manual; however, the mental health staff have removed these two sections. The self-care session was removed, as the discussions included in the START NOW intervention focused on topics inmates at this facility were not able to do while incarcerated, and informal discussions with inmates indicated that this session increased feelings of hopelessness. The session focused on religion and spirituality was also removed after one use, due to initial trials revealing that various group members attempted to use this session as an opportunity to recruit others into their particular religious group.

The second unit the therapist presented in the START NOW intervention, termed $M y$ Emotions: Dealing with Upset Feelings, was comprised of ten sessions and was focused on the ability to understand emotions, recognize emotions, and learn the impact of personal perceptions on responses to emotion. In this unit, therapists taught inmates the following skills: identification of emotions, relation of thoughts and feelings to each other, identification and replacement of 
thought errors (e.g., "expecting the worst"), development of crisis survival skills (e.g., "STOP Skill" to refrain from impulsive actions when emotional), use of distraction techniques for distress tolerance, use of behavioral activation (i.e., pushing themselves to do activities), and identification of anger triggers.

The third unit the therapist presented in the START NOW intervention, termed My Relationships: Building Positive Relationships, consisted of eight sessions and was concentrated on improvement of communication skills such as reception of feedback, assertiveness, requests for support, and development of boundaries (e.g., if you mean "no,” say "no”). In this unit, therapists taught inmates the following skills: development of positive relationships (e.g., address others by name), use of active listening, identification of types of communication styles (i.e., aggressive, assertive, passive), response to feedback, avoidance and implications of the impact of negative relationships (e.g., procriminal friendships), creation and development of personal boundaries (e.g., say "no," when you mean "no"), and acceptance of rejection (e.g., did not get parole).

The final unit the therapist presented in the START NOW intervention, termed $M y$ Future: Setting and Meeting my Goals, included four sessions and was focused on goals for the future. In this unit, therapists taught inmates the following skills: use of positive self-talk regarding the future, goal setting (e.g., request needed information, request support), problemsolving barriers (e.g., submit a formal appeal if disagreeable to a policy), and recognition of positive changes made throughout the program (Sampl, Trestman, \& Krauss, 2013).

The therapists began each START NOW session with a review of the exercises inmates practiced since the last session (Sampl, Trestman, \& Krauss, 2013). This was often completed in a group discussion setting, to provide the opportunity for feedback from other group members 
and therapists. Therapists then moved to the practice and reinforcement of focusing and $\mathrm{ABC}$ skills. Following that, a new skill was introduced to the group. START NOW therapists were encouraged to use motivational interviewing skills to help inmates recognize that they may benefit from the about-to-be-taught skills that would comprise the next unit. After the new skills were taught, there was a group discussion or exercise to review the new skills. Finally, therapists provided inmates with a practice exercise designed to be used between now and the next session to implement the new skill they learned in real-life situations (Sampl, Trestman, \& Krauss, 2013).

Throughout each START NOW session, therapists completed a fidelity checklist. This was implemented $2 / 3$ of the way through the current study, as the mental health staff facilitating these sessions did not previously complete these checklists. In addition to the two abovementioned therapists, a third clinician assisted in completing these checklists. This third clinician was the current Director of Mental Health Services at the correctional facility housing the SCTC, and had extensive experience in working with the START NOW intervention. Fidelity was measured to ensure therapists were adhering to the START NOW treatment manual, but were not utilized for the purpose of improving fidelity in this study.

In addition to participation in the START NOW group sessions, inmates were required to participate in a level system (Roush, Bryson, \& Weaver, 2012). There were four levels in total, and there were specified goals for each level that led to additional privileges. Inmates were observed by mental health staff and correctional officers who both verbally communicated, and completed a log while on the unit regarding inmate behavior. Behaviors required to be promoted to each level, as well as the privileges associated with each level are as follows (Roush, Bryson, \& Weaver, 2012): 
1. Level One: The observation period necessary to reach level one lasts two weeks. Inmates who refrain from self-harm, meet individualized treatment goals, participate in START NOW groups, and do not receive disciplinary infractions will move up to level two. During level one, inmates eat in their cell; can participate in educational classes with permission; can participate in religious services on the SCTC; are provided one hour per day of recreation on the SCTC recreation yard; are unable to receive visits; have a thirty dollar commissary limit; can hold a job on the SCTC; and are required to be escorted by staff to off-unit activities.

2. Level Two: The observation period necessary to reach level two lasts five weeks. Inmates who refrain from self-harm, meet individualized treatment goals, participate in START NOW groups, and do not receive disciplinary infractions will move up to level three. During level two, inmates can eat in the dayroom; can participate in educational classes with permission; can participate in religious services on the SCTC; are provided two hours per day of recreation on the SCTC recreation yard; are allowed two contact visits per month and two non-contact visits per month; have a forty dollar commissary limit; can hold a job on the SCTC; and are required to be escorted by staff to off-unit activities.

3. Level Three: The observation period necessary to reach level three lasts five weeks. Inmates who refrain from self-harm, meet individualized treatment goals, participate in START NOW groups, exhibit appropriate behavior in the general population, and do not receive disciplinary infractions will move up to level four. During level three, inmates can eat in the dayroom; can participate in a variety of classes and activities (i.e., educational classes, church, library, gym); can participate in one religious service off unit 
per week; are provided two and a half hours per day of recreation on the SCTC recreation yard; are allowed four contact visits per month and four non-contact visits per month; have a fifty dollar commissary limit; can hold a job on the SCTC; and after the first week do not need an escort for off-unit activities.

4. Level Four: The observation period necessary to reach level four lasts three weeks. Inmates who refrain from self-harm, meet individualized treatment goals, participate in START NOW groups, exhibit appropriate behavior in general population, and do not receive disciplinary infractions will graduate from the SCTC and return to the general population. During level four, inmates can eat in the dayroom; can participate in all general population activities and recreation; can attend unlimited religious services per week; are able to attend evening recreation time on the general population recreation yard; are allowed eight contact visits per month and eight non-contact visits per month; have a ninety dollar commissary limit; are expected to hold a job in the facility or attend education classes; and do not need an escort for off-unit activities.

If an inmate did not reach the goals set while on a particular level, they were given a warning and if undesirable behavior continued, the treatment team convened and may have extended their time on that particular level or dropped them one level (Roush, Bryson, \& Weaver, 2012). Inmate participation in substance use, trafficking substances, or hurting another inmate automatically led to the drop of a level. If an inmate did not progress (e.g., attend group sessions, engage in group activities), became disruptive, exhibited behavior that required excessive disciplinary infractions, or became a security threat, the treatment team reserved the right to remove that inmate from the SCTC (Roush, Bryson, \& Weaver, 2012).

\section{Outcome Measures}


Participant records at the facility housing the SCTC were accessed by the principal investigator and reviewed through the DCR electronic medical record (CorEMR) and the Offender Identification System (OIS). Due to multiple inmates being incarcerated prior to the use of an electronic medical record in the DCR, some paper charts had to be reviewed. The COVID19 pandemic limited the opportunity to physically go to each of the correctional facilities in the state to obtain these paper charts; therefore, various mental health staff members obtained data from inmate paper chart medical records. Relevant outcome variables needed to evaluate the effectiveness of the START NOW plus level system were abstracted. There were no standardized outcome measures of the START NOW plus level system. Therefore, the outcome measures were as follows: (1) number of referrals to mental health services for misbehavior, (2) number of write-ups in the DCR for each person involved in the START NOW plus level system, (3) number of referrals to the restrictive housing unit, (4) instances of spontaneous use of force, and (5) number of placements on precaution status. These five outcome variables were assessed both 3 months prior to the first session of the START NOW intervention and 3 months following the final session of the START NOW intervention. Three months was chosen to leave enough time for an inmate to be placed in the restrictive housing unit multiple times, as in this particular DCR system, inmates generally receive a 30- or 60-day assignment to restrictive housing. Thus, 3 months would be enough time to denote variability in these outcome variables. Additionally, one previous START NOW investigation utilized a 3-month pre-post design (Shelton \& Wakai, 2011).

Referrals to mental health services were only counted when placed by DCR staff, and when the referral was related to misbehavior. The following did not count toward referrals to 
mental health services: (1) self-referrals, (2) inmates involved in ongoing treatment, or (3) new referrals for treatment for other mental health concerns (outside of misbehavior).

An additional outcome measure in this study includes the number of write-ups for inmate misbehavior or aggressive behavior. For example, write-ups in the WVDCR are defined and divided into classes of severity as follows (WVDCR, 2013):

1. "Class I Offenses: those rule violations that threaten life or limb, which seriously breach facility security and/or public safety or which are felonies" (p. 3). Examples include: Escape, Assault and/or Battery, Rape/Sexual Assault/Sexual Abuse/Sexual Acts, Riot, Arson/Fires, and Hostage Taking

2. "Class II Offenses: those violations which tend to disrupt the normal operation of the institution/facility/center or which subvert institutional systems of control” (p. 9). Examples include: Refusing an Order, Threats, Refusal to Work/Attend Class and Programs, Fighting, and Missing or Confusing Count

3. "Class III Offenses: violations which disturb the normal operation and routine of the institution/facility/center/jail or which manifest a personal problem of adjustment but do not individually rise to a level significant to interfere with parole eligibility" (p. 14). Examples include: Feigning Illness, Littering, Improper Use of Food, Attentiveness, Improper Use of Property, and Proper Clothing

For both Class I and Class II write-ups, inmates may be sanctioned using restrictive housing (WVDCR, 2013). Consistent with this definition of write-ups, the total number of writeups an inmate received in the current study was subtracted from the number of placements they had in the restrictive housing unit to obtain the number of write-ups variable utilized in the current study. This allowed for the outcome variables to be independent, rather than linked with 
each other. The impact of the START NOW plus behavioral level system on inmate misbehavior was assessed by measuring outcome variables 3 months prior to the beginning of and 3 months following completion of the program. Pre-assessment measures did not already exist; therefore, this study included retroactively obtained data through inmate record reviews regarding the time period prior to entering the SCTC. This allowed for assessment of outcome variables both pretreatment and post-treatment.

Inmates can be sanctioned to a restrictive housing unit, pending the severity of a disciplinary infraction. This may be general misbehavior, but can also include aggressive or violent behavior. The National Commission on Correctional Health Care (2016) has compiled the reasons in which most correctional facilities make restrictive housing referrals. These reasons are as follows:

1. Minor rule breaking behavior (e.g., failure to obey order)

2. Behavior that may create safety or security concerns (e.g., gang membership)

3. Protective custody (e.g., transgender individuals)

4. Clinical/therapeutic reasons (e.g., mental health concerns that create difficulty for inmates to adhere to facility policies)

Additionally, when inmates exhibit behavior that may be viewed as a threat to the safety and security of a correctional institution, a use of force may occur. For example, the Oklahoma Department of Corrections supplies their spontaneous use of force policy to the public. The following definition and information regarding spontaneous use of force was based on the policy in the Oklahoma Department of Corrections (ODOC, 2018).

Use of force by correctional officers is defined by physical contact, fire power, chemical agents, or inflammatory agents necessary to control inmate behavior and/or maintain security 
and safety (e.g., avoid an escape, self-defense to avoid bodily harm) in the facility. However, physical force is not to be used excessively or as a punishment. Examples of items to assist in carrying out use of force are weapons, physical restraints (e.g., handcuffs), Oleoresin Capsicum (i.e., OC or pepper spray), tasers, and projectile devices (e.g., pepper ball; ODOC, 2018).

The final outcome variable utilized in the current study was precaution status (i.e., suicide watch). Inmates who are considered acutely suicidal (i.e., actively engaging in self-injurious behavior or exhibiting suicidal ideation/intent with a specific plan) are monitored by staff via constant observation (National Commission on Correctional Health Care, 2018). Inmates who are nonacutely suicidal (i.e., exhibiting suicidal ideation without a specific plan) are monitored by staff at varied intervals, with no longer than 15 minutes between each check. Non-mental health staff may initially place an inmate on precaution status; however, an evaluation by a mental health professional is required to determine the level of risk, supervision, and need for mental health services for that particular inmate. Inmates on precaution status are reassessed regularly to determine changes in behavior that may impact the necessary supervision level. Inmates on precaution status are housed closely to staff, and property is restricted to ensure there are no items in their cell that may assist in a suicide attempt (e.g., sheets that would enable hanging; National Commission on Correctional Health Care, 2018).

Variables obtained through the evaluation included inmate DCR number (assigned to each particular inmate involved in the SCTC), reason for restrictive housing referral, and date the inmate was placed in the restrictive housing unit. This allowed for comprehensive comparisons prior to and following the completion of the program. Additional demographic data was gathered through the electronic medical records (CorEMR) that might impact the effectiveness of the START NOW program (i.e., age, education level, race/ethnicity, number of mental health 
diagnoses, number of days in prison before START NOW participation, and number of START NOW sessions attended).

\section{Results}

Prior to running any statistical analyses, missingness, normality, and outliers were assessed. The mental health referral variable was the only outcome variable with missing data. There were 10 participants (11.4\%) with missing data for pre-intervention mental health referrals and 8 participants $(9.1 \%)$ with missing data for post-intervention mental health referrals. However, this missingness was due to feasibility, as a portion of participants' paper charts were unable to be located during the duration of the study. A floor effect was present in the three outcome variables of mental health referrals, precaution status, and use of force, thereby precluding the use of ANOVAs. Therefore, chi-square analyses were run on these variables.

Skew values were less than 3.0 for all outcome variables, with the exception of the control group pre-intervention restrictive housing variable $($ skew $=4.42, S E=.38)$. The following three outcome variables had kurtosis values above 3.0: (1) START NOW + BLS group post-intervention restrictive housing (kurtosis $=5.46, S E=.78$ ), (2) control group preintervention restrictive housing (kurtosis $=16.27, S E=.75$ ), and (3) START NOW + BLS group post-intervention write-ups (kurtosis $=4.17, S E=.78)$. The variables with high skew and kurtosis are likely a result of the following: (1) the number of times a participant was able to be placed in restrictive housing was limited (range $0-3$, but most frequently was 1 ), (2) the matched control group, which was required to have been placed in restrictive housing during the same three-month period as a START NOW + BLS participant, and (3) the number of write-ups a participant received was limited (1-3 was most common, and 1 was the most frequent number of write-ups received). 
To ensure that ANOVA assumptions were not violated, extreme outliers on all outcome variables were examined. Pre-intervention and post-intervention write-ups were the only outcome variables in which extreme outliers were present. Three extreme outliers were removed prior to running a repeated-measures ANOVA for number of write-ups. Since the START NOW + BLS and control group participants were matched, the corresponding matches to the three outliers were also removed. The following are the scores of the six removed participants: (1) preintervention 3 write-ups, post-intervention 13 write-ups; (2) pre-intervention 10 write-ups, postintervention 2 write-ups; (3) pre-intervention 0 write-ups, post-intervention 9 write-ups; (4) preintervention 3 write-ups, post-intervention 1 write-up; (5) pre-intervention 3 write-ups, postintervention 1 write-up; and (6) pre-intervention and post-intervention 0 write-ups.

\section{Adherence to the START NOW Intervention}

To determine if these results were a true representation of the integrity of the treatment, fidelity checks included in the START NOW facilitator manual were completed by a group therapist and obtained for one round of the START NOW program. These forms include two sections: content and process. The content section evaluates whether the therapist covered the particular session's content "fully, somewhat, or not at all." It also requires a rating on a scale from 0 to $5(0=$ Not Covered, $1=$ Very Ineffective, $2=$ Ineffective, $3=$ Acceptable, $4=$ Effective, and $5=$ Very Effective). The process section evaluates whether the therapist demonstrated these fully, somewhat, or not at all. Similar to the content section, the process section requires ratings on the same 0 to 5 scale. A majority of the START NOW sessions (21 out of 27) were conducted by one therapist. Overall, the therapists delivered the content and process of the START NOW treatment fully, $M=1.61, S D=.57,80.8 \%$ and effectively, $M=$ $3.99, S D=.90,79.8 \%$. There was similar adherence between the two therapists. They both 
completed the sessions fully, $M=1.60, S D=.57,80.1 \%$ versus $M=1.66, S D=.57,82.9 \%$ and effectively, $M=3.98, S D=1.01,79.6 \%$ versus $M=4.03, S D=.34,80.6 \%$.

\section{Preliminary Analyses}

Pearson's product-moment correlations were used to determine the association between participant characteristics (i.e., demographics, offense type, time to parole, number of START NOW sessions completed, highest level obtained in the BLS, graduation from the SCTC, and mental health diagnoses), and all outcome variables (i.e., mental health referrals, use of force, precaution status, restrictive housing, and write-ups pre-intervention and post-intervention). Gender and ethnicity variables were not included in analyses, as all participants were male and non-Hispanic. Race, age, and offense type were not associated with any outcome variables preintervention or post-intervention. Education level was negatively associated with precaution status pre-intervention $(r(88)=-.28, p=.008)$.

Various correlations between outcome variables, demographics, and other participant characteristics (e.g., time to parole, mental health diagnoses) were run on the total number of participants. Total number of sessions attended was positively associated with use of force preintervention $(r(88)=.23, p=.029)$, use of force post-intervention $(r(88)=.25, p=.018)$, and precaution status pre-intervention $(r(88)=.27, p=.011)$. Graduation status (graduated or did not graduate from the SCTC) was negatively associated with use of force post-intervention $(r(45)=$ $.31, p=.039)$, restrictive housing post-intervention $(r(45)=-.42, p=.004)$, and precaution status post-intervention $(r(45)=-.36, p=.017)$. Highest level attained in the SCTC was positively associated with graduation status $(r(45)=.83, p<.001)$, and negatively associated with use of force post-intervention $(r(45)=-.39, p=.008)$, restrictive housing post-intervention $(r(45)=$ $.45, p=.002)$, precaution status pre-intervention $(r(45)=-.50, p<.001)$, and precaution status 
post-intervention $(r(45)=-.42, p=.004)$. Time to parole was negatively associated with highest level attained in the SCTC $(r(45)=-.45, p=.002)$ and total sessions attended $(r(88)=-.36, p=$ $.001)$.

Participant mental health diagnoses were divided into three categories: (1) personality disorder, (2) impulse control disorder, and (3) substance use disorder. Participant type (START NOW + BLS versus control group) was negatively associated with diagnosis of a personality disorder $(r(88)=-.25, p=.019)$. Diagnosis of a personality disorder was positively associated with diagnosis of an impulse control disorder $(r(88)=.45, p<.001)$, diagnosis of a substance use disorder $(r(88)=.27, p=.010)$, and use of force post-intervention $(r(88)=.23, p=.028)$.

Diagnosis of an impulse control disorder was positively associated with diagnosis of a substance use disorder $(r(88)=.33, p=.002)$, mental health referrals pre-intervention $(r(78)=.23, p=$ $.039)$, and use of force post-intervention $(r(88)=.24, p=.025)$. For additional information regarding correlation analyses, see table 2 .

\section{Pre-Intervention Period}

All pre-intervention analyses were run utilizing the entire number of participants who completed at least 17 sessions of the START NOW + BLS group and their matched control. As a reminder, 17 or more sessions were selected because Cislo and Trestman (2016) found improvements in START NOW outcome variables up until completion of 17 sessions. In the current study, the inclusion of inmates who completed 17 or more START NOW sessions also allowed for a larger sample size to provide adequate power for statistical analyses.

Chi-Square Analyses. A chi-square analysis was conducted between group membership (i.e., START NOW + BLS or control group) and mental health referrals. There was not a statistically significant association between group membership and mental health referrals pre- 
intervention, $\chi^{2}(1)=2.093, p=.148$. A chi-square analysis was conducted between group membership (i.e., START NOW + BLS or control group) and use of force. There was not a statistically significant association between group membership and use of force pre-intervention, $\chi^{2}(1)=.205, p=.651$. A chi-square analysis was conducted between group membership (i.e., START NOW + BLS or control group) and precaution status. There was a statistically significant association between group membership and precaution status pre-intervention, $\chi^{2}(1)=$ $4.657, p=.031$. In the START NOW + BLS group, 7 participants were placed on precaution status in the pre-intervention period, while only 1 participant in the control group was placed on precaution status in the pre-intervention period.

T-Tests. An independent samples t-test was run to determine if there were differences between the START NOW + BLS group and the control group on pre-intervention placement in restrictive housing. There was no significant difference between the START NOW + BLS group $(M=1.20, S D=.66)$ and the control group $(M=1.14, S D=.41), t(74.33)=.517, p=.606$.

An independent samples t-test was run to determine if there were differences between the START NOW + BLS group and the control group on pre-intervention number of write-ups. There was a significant difference between the START NOW + BLS group $(M=1.57, S D=$ $1.86)$ and the control group $(M=.80, S D=1.22), t(71.20)=2.23, p=.029$.

\section{Post-Intervention Period}

All post-intervention analyses were run utilizing the entire number of participants who completed at least 17 sessions of the START NOW + BLS group and their matched control.

Chi-Square Analyses. Given that a minimal number of participants in the postintervention period received a mental health referral $(n=5)$, were involved in a use of force $(n=$ $8)$, or were placed on precaution status $(n=8)$, various chi-square analyses were used as the 
method of analysis. Since chi-square analyses do not allow for within-subjects design, each postintervention chi-square analysis was compared with a pre-intervention chi-square analysis, which was run prior to the parallel post-intervention chi-square analysis to determine whether there were any differences between the groups in the pre-intervention period. Thus, if postintervention differences did exist, they could be attributed to the SCTC. For information regarding descriptive statistics, see table 1.

A chi-square analysis was conducted between group membership (i.e., START NOW + BLS or control group) and mental health referrals. There was not a statistically significant association between group membership and mental health referrals post-intervention, $\chi^{2}(1)=$ $1.920, p=.166$. A chi-square analysis was conducted between group membership (i.e., START NOW + BLS or control group) and use of force. There was not a statistically significant association between group membership and use of force post-intervention, $\chi^{2}(1)=.455, p=.500$. A chi-square analysis was conducted between group membership (i.e., START NOW + BLS or control group) and precaution status. There was not a statistically significant association between group membership and precaution status post-intervention, $\chi^{2}(1)=2.006, p=.157$.

Restrictive Housing ANOVA. A repeated-measures ANOVA (pre-intervention versus post-intervention x START NOW + BLS versus control) was run to determine the impact of the group membership on the number of times participants were placed in restrictive housing during the pre-intervention and post-intervention period (i.e., START NOW + BLS or control group). There were no outliers, and although the data were not normally distributed, both levels of the independent variable (START NOW + BLS versus control group) were similarly skewed kurtotic. Therefore, lack of normality should not impact Type I error rate substantially. 
Generally, a repeated-measures ANOVA is considered robust to data that is not normally distributed.

There was a significant main effect of time for restrictive housing pre-intervention to post-intervention $\left(F(1,86)=40.420, p=.000\right.$, partial $\eta^{2}=.320 ; M$ Time 1 $=1.17, S E$ Time $1=.06$ versus $M_{\text {Time } 2}=.58, S E_{\text {Time } 2}=.08$ ). The main effect of group membership on restrictive housing was not significant $\left(F(1,86)=.074, p=.786\right.$, partial $\eta^{2}=.001 ; M$ START NOW + BLS group $=.89, S E$ START NOW + BLS group $=.07$ versus $M$ control group $=.86, S E$ control group $=.08)$. There was not a statistically significant interaction between group membership and placement in restrictive housing, $F(1,86)$ $=.119, p=.731$, partial $\eta^{2}=.001$, with number of restrictive housing placements decreasing from pre-intervention $(M=1.20, S D=.66)$ to post-intervention $(M=.58, S D=.72)$ for the START NOW + BLS group, and decreasing from pre-intervention $(M=1.14, S D=.41)$ to postintervention $(M=.58, S D=.76)$ for the control group. For additional information regarding descriptive statistics, see table 1.

However, due to the limited variance $($ minimum $=0$, maximum $=3$ ) of placement in restrictive housing, a chi-square analysis was run to evaluate any significant differences. A chisquare analysis was conducted between placement in restrictive housing and group membership (i.e., START NOW + BLS or control group). There was not a statistically significant association between restrictive housing post-intervention and group membership, $\chi^{2}(1)=.055, p=.815$.

Write-ups ANOVA. A repeated-measures ANOVA was run to determine the impact of group membership (i.e., START NOW + BLS or control group) on pre- and post-intervention number of write-ups. Although the data were not normally distributed, both levels of the independent variable (START NOW + BLS versus control group) are similarly skewed. 
Therefore, lack of normality should not impact Type I error rate substantially. Generally, a repeated-measures ANOVA is considered robust to data that is not normally distributed.

Due to the significant difference between the START NOW + BLS group and control group on pre-intervention write-ups, an ANCOVA utilizing pre-intervention write-ups as a covariate was run. The ANCOVA utilizing this as a covariate was not significant; therefore, the original repeated-measures ANOVA (pre-intervention versus post-intervention x START NOW

+ BLS versus control) is reported here. There was not a significant main effect of time for writeups pre-intervention to post-intervention $\left(F(1,80)=3.071, p=.084\right.$, partial $\eta^{2}=.037 ; M_{\text {Time } 1}=$ $1.19, S E_{\text {Time } 1}=.18$ versus $\left.M_{\text {Time } 2}=.81, S E_{\text {Time } 2}=.16\right)$. The main effect of group membership on write-ups was significant $\left(F(1,80)=7.850, p=.006\right.$, partial $\eta^{2}=.089 ; M$ START NOW + BLS group $=$ $1.36, S E$ START NOW + BLS group $=.18$ versus $M$ control group $=.64, S E$ control group $=.18$ ). There was not a statistically significant interaction between group membership and number of write-ups, $F(1,80)$ $=.058, p=.810$, partial $\eta^{2}=.001$, with number of write-ups decreasing from pre-intervention $(M$ $=1.57, S D=1.86)$ to post-intervention $(M=1.14, S D=1.82)$ for the START NOW + BLS group, and decreasing from pre-intervention $(M=.80, S D=1.22)$ to post-intervention $(M=.48$, $S D=.91$ ) for the control group. For additional information regarding descriptive statistics, see table 1 .

\section{Mental Health Diagnoses}

Due to the multiple significant correlations between outcome variables in the postintervention period and various mental health diagnosis categories (i.e., personality disorders, impulse control disorders, and substance use disorders), six additional exploratory ANOVAs utilizing these diagnosis categories were run on the full sample of participants who completed 17 or more sessions of the START NOW intervention. 
Personality Disorders. A repeated-measures ANOVA was run to determine the impact of group membership (i.e., START NOW + BLS or control group) and personality disorder (i.e., at least one, $n=44$ or no diagnosis, $n=44$ ) on pre-intervention and post-intervention placement in restrictive housing. There was a significant main effect of time for restrictive housing preintervention to post-intervention $\left(F(1,84)=35.919, p<.001\right.$, partial $\eta^{2}=.300 ; M$ Time $1=1.17$, $S E_{\text {Time } 1}=.06$ versus $\left.M_{\text {Time } 2}=.59, S E_{\text {Time } 2}=.08\right)$. The main effect of group membership on restrictive housing was not significant $\left(F(1,84)=.011, p=.916\right.$, partial $\eta^{2}=.000 ; M$ START NOW + BLS group $=.89, S E$ START NOW + BLS group $=.08$ versus $M_{\text {control group }}=.88, S E$ control group $\left.=.08\right)$. The main effect of personality disorder diagnosis on restrictive housing was not significant $(F(1,84)=$ $.375, p=.542$, partial $\eta^{2}=.004 ; M$ personality disorder $=.92, S E_{\text {personality disorder }}=.08$ versus $M$ no personality disorder $=.85, S E$ no personality disorder $=.08)$. There was not a statistically significant interaction between group membership, personality disorder diagnosis, and placement in restrictive housing, $F(1,84)$ $=.144, p=.705$, partial $\eta^{2}=.002$, with number of restrictive housing placements for those participants with personality disorders decreasing from pre-intervention $(M=1.21, S D=.69)$ to post-intervention $(M=.57, S D=.69)$ for the START NOW + BLS group, and decreasing from pre-intervention $(M=1.19, S D=.40)$ to post-intervention $(M=.69, S D=.95)$ for the control group. Additionally, number of restrictive housing placements for those participants without personality disorders decreased from pre-intervention $(M=1.18, S D=.64)$ to post-intervention $(M=.59, S D=.80)$ for the START NOW + BLS group, and decreased from pre-intervention $(M$ $=1.11, S D=.42)$ to post-intervention $(M=.52, S D=.64)$ for the control group.

A repeated-measures ANOVA was run to determine the impact of group membership (i.e., START NOW + BLS or control group) and personality disorder (i.e., at least one, $n=44$ or no diagnosis, $n=44$ ) on pre-intervention and post-intervention number of write-ups. There was a 
significant main effect of time for write-ups pre-intervention to post-intervention $(F(1,78)=$ 4.098, $p=.046$, partial $\eta^{2}=.050 ; M_{\text {Time } 1}=1.24, S E_{\text {Time 1 }}=.18$ versus $M$ Time 2 $=.79, S E_{\text {Time 2 }}=$ .17). The main effect of group membership on write-ups was significant $(F(1,78)=5.353, p=$ .023, partial $\eta^{2}=.064 ; M$ START NOW + BLS group $=1.32, S E$ START NOW + BLS group $=.18$ versus $M$ control group $=$ $.71, S E$ control group $=.19)$. The main effect of personality disorder diagnosis on write-ups was not significant $\left(F(1,78)=2.957, p=.089\right.$, partial $\eta^{2}=.037 ; M_{\text {personality disorder }}=1.24, S E_{\text {personality disorder }}=$ .19 versus $M$ no personality disorder $=.79, S E$ no personality disorder $=.19)$. There was not a statistically significant interaction between group membership, personality disorder diagnosis, and number of write-ups, $F(1,78)=1.763, p=.188$, partial $\eta^{2}=.022$, with number of write-ups for those participants with personality disorders decreasing from pre-intervention $(M=1.62, S D=1.81)$ to post-intervention $(M=1.35, S D=1.94)$ for the START NOW + BLS group, and decreasing from pre-intervention $(M=1.40, S D=1.45)$ to post-intervention $(M=.60, S D=1.24)$ for the control group. Additionally, number of write-ups for those participants without personality disorders decreased from pre-intervention $(M=1.50, S D=2.00)$ to post-intervention $(M=.81$, $S D=1.60)$ for the START NOW + BLS group, and decreased from pre-intervention $(M=.44$, $S D=.92)$ to post-intervention $(M=.40, S D=.65)$ for the control group.

Impulse Control Disorders. A repeated-measures ANOVA was run to determine the impact of group membership (i.e., START NOW + BLS or control group) and impulse control disorder (i.e., at least one, $n=33$ or no diagnosis, $n=55$ ) on pre-intervention and postintervention placement in restrictive housing. There was a significant main effect of time for restrictive housing pre-intervention to post-intervention $\left(F(1,84)=33.873, p=.000\right.$, partial $\eta^{2}=$ $.287 ; M_{\text {Time } 1}=1.18, S E_{\text {Time } 1}=.06$ versus $\left.M_{\text {Time } 2}=.60, S E_{\text {Time } 2}=.09\right)$. The main effect of group membership on restrictive housing was not significant $\left(F(1,84)=.036, p=.851\right.$, partial $\eta^{2}=$ 
$.000 ; M$ StART NOW + BLS group $=.88, S E$ START NOW + BLS group $=.07$ versus $M$ control group $=.90, S E$ control group $=$ .08). The main effect of impulse control disorder diagnosis on restrictive housing was not significant $\left(F(1,84)=.000, p=1.000\right.$, partial $\eta^{2}=.000 ; M$ impulse control disorder $=.89, S E$ impulse control disorder $=.09$ versus $M$ no impulse control disorder $=.89, S E$ no impulse control disorder $=.07)$. There was not a statistically significant interaction between group membership, impulse control disorder diagnosis, and placement in restrictive housing, $F(1,84)=.068, p=.794$, partial $\eta^{2}=.001$, with number of restrictive housing placements for those participants with impulse control disorders decreasing from pre-intervention $(M=1.05, S D=.50)$ to post-intervention $(M=.52, S D=.81)$ for the START NOW + BLS group, and decreasing from pre-intervention $(M=1.25, S D=.45)$ to post-intervention $(M=.75, S D=.97)$ for the control group. Additionally, number of restrictive housing placements for those participants without impulse control disorders decreased from preintervention $(M=1.33, S D=.76)$ to post-intervention $(M=.63, S D=.65)$ for the START NOW + BLS group, and decreased from pre-intervention $(M=1.10, S D=.40)$ to post-intervention $(M$ $=.52, S D=.68)$ for the control group.

A repeated-measures ANOVA was run to determine the impact of group membership (i.e., START NOW + BLS or control group) and impulse control disorder (i.e., at least one, $n=$ 33 or no diagnosis, $n=55$ ) on pre-intervention and post-intervention number of write-ups. There was not a significant main effect of time for write-ups pre-intervention to post-intervention $(F(1$, $78)=3.809, p=.055$, partial $\eta^{2}=.047 ; M_{\text {Time } 1}=1.31, S E_{\text {Time } 1}=.19$ versus $M_{\text {Time } 2}=.85, S E$ Time $2=.17)$. The main effect of group membership on write-ups was significant $(F(1,78)=4.568, p$ $=.036$, partial $\eta^{2}=.055 ; M$ StART NOW + BLS group $=1.37, S E$ START NOW + BLS group $=.18$ versus $M$ control group $=.79, S E$ control group $=.21$ ). The main effect of impulse control disorder diagnosis on write-ups was significant $\left(F(1,78)=5.466, p=.022\right.$, partial $\eta^{2}=.065 ; M$ impulse control disorder $=1.40, S E$ impulse control 
disorder $=.22$ versus $M$ no impulse control disorder $=.76, S E$ no impulse control disorder $=.16)$. There was not a statistically significant interaction between group membership, impulse control disorder diagnosis, and number of write-ups, $F(1,78)=.281, p=.598$, partial $\eta^{2}=.004$, with number of write-ups for those participants with impulse control disorders decreasing from pre-intervention $(M=1.95, S D=2.24)$ to post-intervention $(M=1.45, S D=2.04)$ for the START NOW + BLS group, and decreasing from pre-intervention $(M=1.50, S D=1.58)$ to post-intervention $(M=.70$, $S D=1.25$ ) for the control group. Additionally, number of write-ups for those participants without impulse control disorders decreased from pre-intervention $(M=1.23, S D=1.41)$ to postintervention $(M=.86, S D=1.58)$ for the START NOW + BLS group, and decreased from preintervention $(M=.57, S D=1.01)$ to post-intervention $(M=.40, S D=.77)$ for the control group.

Substance Use Disorders. A repeated-measures ANOVA was run to determine the impact of group membership (i.e., START NOW + BLS or control group) and substance use disorder (i.e., at least one, $n=48$ or no diagnosis, $n=40$ ) on pre-intervention and post-intervention placement in restrictive housing. There was a significant main effect of time for restrictive housing pre-intervention to post-intervention $\left(F(1,84)=37.836, p=.000\right.$, partial $\eta^{2}=.311 ; M$ Time 1 $=1.18, S E_{\text {Time } 1}=.06$ versus $\left.M_{\text {Time } 2}=.59, S E_{\text {Time } 2}=.08\right)$. The main effect of group membership on restrictive housing was not significant $\left(F(1,84)=.109, p=.742\right.$, partial $\eta^{2}=$ $.001 ; M$ StART NOW + BLS group $=.90, S E$ START NOW + BLS group $=.08$ versus $M$ control group $=.86, S E$ control group $=$ .08). The main effect of substance use disorder diagnosis on restrictive housing was not significant $\left(F(1,84)=.004, p=.948\right.$, partial $\eta^{2}=.000 ; M$ substance use disorder $=.88, S E$ substance use disorder $=$ .07 versus $M$ no substance use disorder $\left.=.89, S E_{\text {no substance use disorder }}=.08\right)$. There was not a statistically significant interaction between group membership, substance use disorder diagnosis, and placement in restrictive housing, $F(1,84)=.117, p=.733$, partial $\eta^{2}=.001$, with number of 
restrictive housing placements for those participants with substance use disorders decreasing from pre-intervention $(M=1.21, S D=.56)$ to post-intervention $(M=.52, S D=.74)$ for the START NOW + BLS group, and decreasing from pre-intervention $(M=1.26, S D=.56)$ to postintervention $(M=.53, S D=.90)$ for the control group. Number of restrictive housing placements for those participants without substance use disorders decreased from pre-intervention $(M=1.19$, $S D=.83)$ to post-intervention $(M=.69, S D=.70)$ for the START NOW + BLS group, and decreased from pre-intervention $(M=1.04, S D=.20)$ to post-intervention $(M=.63, S D=.65)$ for the control group, but neither of these differences reached statistical significance.

A repeated-measures ANOVA was run to determine the impact of group membership (i.e., START NOW + BLS or control group) and substance use disorder (i.e., at least one, $n=44$ or no diagnosis, $n=38$ ) on pre-intervention and post-intervention number of write-ups. Due to the significant difference between the START NOW + BLS group and control group on preintervention write-ups, an ANCOVA utilizing pre-intervention write-ups as a covariate was run. The ANCOVA utilizing this as a covariate was not significant; therefore, the original repeated measures ANOVA is reported here. There was a significant main effect of time for write-ups pre-intervention to post-intervention $\left(F(1,78)=5.606, p=.020\right.$, partial $\eta^{2}=.067 ; M_{\text {Time } 1}=$ $1.29, S E_{\text {Time } 1}=.18$ versus $M$ Time $\left.2=.77, S E_{\text {Time } 2}=.17\right)$. The main effect of group membership on write-ups was significant $\left(F(1,78)=5.853, p=.018\right.$, partial $\eta^{2}=.070 ; M$ START NOW + BLS group $=$ $1.35, S E$ StART NOW + BLS group $=.19$ versus $M$ control group $=.71, S E$ control group $=.19)$. The main effect of substance use disorder diagnosis on write-ups was not significant $(F(1,78)=2.074, p=.154$, partial $\eta^{2}=.026 ; M$ substance use disorder $=.1 .22, S E$ substance use disorder $=.18$ versus $M$ no substance use disorder $=.84$, $S E$ no substance use disorder $=.19$ ). There was a statistically significant interaction between group membership, substance use disorder diagnosis, and number of write-ups, $F(1,84)=4.681, p=$ 
.034 , partial $\eta^{2}=.057$, with number of write-ups for those participants without substance use disorders decreasing from pre-intervention $(M=2.00, S D=2.35)$ to post-intervention $(M=.64$, $S D=1.45)$ for the START NOW + BLS group, and decreasing from pre-intervention $(M=.42$, $S D=.83)$ to post-intervention $(M=.29, S D=.69)$ for the control group. The number of writeups for those participants with substance use disorders increased from pre-intervention $(M=$ $1.36, S D=1.57)$ to post-intervention $(M=1.39, S D=1.95)$ for the START NOW + BLS group, and decreased from pre-intervention $(M=1.38, S D=1.50)$ to post-intervention $(M=.75, S D=$ 1.13) for the control group.

\section{Graduates Versus Non-Graduates of the SCTC}

To ensure that the lack of significant results was not a function of including data from participants who completed at least 17 START NOW sessions rather than including data from participants who completed the START NOW intervention. ANOVAs were run including only graduates of the SCTC and their matched control. Graduates of the SCTC completed the entirety of the START NOW + BLS intervention.

Restrictive Housing ANOVA. A repeated-measures ANOVA was run to determine the impact of group membership (i.e., START NOW + BLS, $n=27$ or control group, $n=27$ ) on preintervention and post-intervention placement in restrictive housing. There was a significant main effect of time for restrictive housing pre-intervention to post-intervention $(F(1,52)=38.172, p=$ .000, partial $\eta^{2}=.423 ; M_{\text {Time } 1}=1.19, S E_{\text {Time } 1}=.07$ versus $M$ Time $2=.46, S E$ Time $\left.2=.10\right)$. The main effect of group membership on restrictive housing was not significant $(F(1,52)=1.822, p$ $=.183$, partial $\eta^{2}=.034 ; M$ stART NOW + BLS group $=.74, S E$ START NOW + BLS group $=.09$ versus $M_{\text {control group }}=$ $.91, S E$ control group $=.09)$. There was not a statistically significant interaction between group membership and placement in restrictive housing, $F(1,52)=.627, p=.432$, partial $\eta^{2}=.012$, 
with number of restrictive housing placements decreasing from pre-intervention $(M=1.15, S D=$ $.46)$ to post-intervention $(M=.33, S D=.68)$ for the START NOW + BLS group, and decreasing from pre-intervention $(M=1.22, S D=.51)$ to post-intervention $(M=.59, S D=.80)$ for the control group.

Write-ups $A N O V A$. A repeated-measures ANCOVA was run to determine the impact of group membership (i.e., START NOW + BLS, $n=25$ or control group, $n=25$ ) on pre- and postintervention number of write-ups. Due to the significant difference between the START NOW + BLS group and control group on pre-intervention write-ups, an ANCOVA utilizing preintervention write-ups as a covariate was run. The ANCOVA utilizing this as a covariate was not significant; therefore, the original repeated-measures ANOVA is reported here. There was a significant main effect of time for write-ups pre-intervention to post-intervention $(F(1,48)=$ 4.322, $p=.043$, partial $\eta^{2}=.083 ; M_{\text {Time 1 }}=1.24, S E$ Time $1=.22$ versus $M$ Time $2=.66, S E$ Time $2=$ .21). The main effect of group membership on restrictive housing was not significant $(F(1,48)=$ $3.792, p=.057$, partial $\eta^{2}=.073 ; M$ START NOW + BLS group $=1.26, S E$ START NOW + BLS group $=.23$ versus $M$ control group $=.64, S E$ control group $=.23)$. There was not a statistically significant interaction between group membership and number of write-ups, $F(1,48)=.869, p=.356$, partial $\eta^{2}=.018$, with number of write-ups decreasing from pre-intervention $(M=1.68, S D=1.86)$ to post-intervention $(M=.84, S D=1.84)$ for the START NOW + BLS group, and decreasing from pre-intervention $(M=.80, S D=1.12)$ to post-intervention $(M=.48, S D=.92)$ for the control group.

\section{Discussion}

The current study sought to examine the impact of the START NOW intervention plus a behavioral level system (BLS) in a self-contained therapeutic community (SCTC) on inmate misbehavior at a correctional facility in a southern state. Although multiple studies have 
previously evaluated the START NOW intervention, the weaknesses and limitations of these previous designs suggested the need for additional research regarding the START NOW intervention. Some of these limitations included descriptive approaches to analyzing data, small sample sizes, analysis of post-treatment data only, and a lack of focus on participants who exhibit largely violent, aggressive, and disruptive behavior while incarcerated (Cislo \& Trestman, 2016; Kersten, Cislo, Lynch, Shea, \& Trestman 2015; Shelton \& Wakai, 2011). To improve the evidence base for the START NOW intervention, this study (1) evaluated the intervention with a sample of inmates exhibiting violent, aggressive, and disruptive behavior while incarcerated and (2) utilized a stronger design that included a pre-treatment to posttreatment design with a control group.

In contrast to previous studies (Cislo \& Trestman, 2016; Kersten, Cislo, Lynch, Shea, \& Trestman 2015; Shelton \& Wakai, 2011), the current study, with the inclusion of a control group as well as pre-intervention to post-intervention data comparison, showed largely nonsignificant results. Chi-square analyses post-intervention were all nonsignificant, indicating that the START NOW + BLS group was not significantly different from the control group on the outcomes of placement in restrictive housing, instances of spontaneous use of force, number of mental health referrals, and placement on precaution status. The ANCOVA for the write-up variable as the outcome variable, including group membership (i.e., START NOW + BLS or control group) as the independent variable, and a covariate of total sessions attended was not significant. The ANCOVA for the restrictive housing variable as the outcome variable, including group membership (i.e., START NOW + BLS or control group) as the independent variable, and a covariate of total sessions attended was also not significant. There was a main effect of time (pre-intervention to post-intervention) for both the write-up and restrictive housing variables 
with no significant interaction effects, thereby demonstrating that the participants in the START NOW + BLS group improved behaviorally from pre-intervention to post-intervention, but at a rate similar to the participants in the control group. This is not likely due to lack of adherence to the START NOW treatment manual, as data showed therapists were delivering the content and process of the START NOW treatment fully and effectively.

As a participant's time to parole decreased, the highest level attained in the SCTC and number of START NOW sessions attended increased. Therefore, as inmates approach their parole eligibility date, they may become more motivated to engage in the START NOW intervention and behavioral level system. Diagnosis of a personality disorder or impulse control disorder was associated with an increase in use of force post-intervention. Thus, mental health diagnoses may impact improvement in behavioral dysregulation post-intervention. Although there were significant correlations between some participant characteristics (e.g., time to parole) and either treatment outcome variables or treatment outcome results, it was not always possible to further divide the groups (i.e., START NOW + BLS versus control) into subgroups due to sample size, thereby making it impossible to determine the impact of these associations on START NOW treatment outcomes.

Despite previous investigations which found that inmates spent fewer days in a mental health unit following completion of the START NOW intervention (Shelton \& Wakai, 2011), the current study did not find a significant reduction in mental health referrals from pre-intervention to post-intervention in the START NOW + BLS group compared with the control group. Previous studies also found a reduction in the number of write-ups for misbehavior following completion of the START NOW treatment (Shelton \& Wakai, 2011; Kersten, Cislo, Lynch, Shea, \& Trestman, 2015); however, the current study did not find a significant reduction in the 
number of write-ups from pre-intervention to post-intervention in the START NOW + BLS group compared with the control group. Additionally, one previous study found that inmates who were deemed a higher security risk experienced the greatest decrease in disciplinary reports (i.e., write-ups) following the START NOW intervention (Kersten, Cislo, Lynch, Shea, \& Trestman 2015). Although the current study evaluated this program with a sample of inmates who were considered to have high levels of aggressive and disruptive behavior, there was not a significant decrease in number write-ups, despite previous START NOW research indicating a greater decrease in disciplinary reports for inmates who were deemed a higher security risk.

Finally, previous investigations found that inmates spent fewer days in restrictive housing following completion of the START NOW intervention (Shelton \& Wakai, 2011). We hypothesized that this finding would extend to the number of placements in the restrictive housing unit. However, the current study did not find a significant reduction in the number of placements in restrictive housing from pre-intervention to post-intervention in the START NOW + BLS group compared to the control group.

No hypotheses were made regarding the impact of the START NOW intervention on the number of instances of spontaneous use of force, as previous investigations had not examined this variable. Similarly to other outcome variables, there was not a significant reduction in instances of spontaneous use of force from pre-intervention to post-intervention in the START NOW + BLS group compared to the control group.

Additional analyses were run to evaluate the impact of type of mental health disorder (i.e., personality disorder, impulse control disorder, and substance use disorder) on outcome variables. There was a statistically significant interaction between group membership, substance use disorder diagnosis, and number of write-ups. The number of write-ups for those participants 
without substance use disorders decreased from pre-intervention to post-intervention for the START NOW + BLS group and for the control group. However, the number of write-ups for those participants with substance use disorders increased from pre-intervention to postintervention for the START NOW + BLS group and decreased for the control group. Although this finding does indicate the need for further research regarding the success of the START NOW program with participants who have a significant substance use history, multiple ANOVAs were run in this study; thus, this finding should be interpreted with caution. Moreover, without further information about each participant (e.g., the date each participant was diagnosed in proximity to their entrance into the SCTC), it is difficult to devise any explanations regarding this finding.

These findings (i.e., lack of significant outcomes suggesting that the START NOW + BLS intervention reduces behavioral dysregulation compared with a control group) are in contrast to previous research evaluating the START NOW intervention. However, previous studies utilized significantly different methodologies from the current study. Specifically, previous studies did not include a comparison group that had not received the START NOW intervention, nor did these studies statistically evaluate outcome variables from pre-intervention to post-intervention. Although the inmates involved in the START NOW + BLS intervention displayed a decrease in the outcome variables associated with behavioral dysregulation, the inmates in the control group also made improvements from pre-intervention to post-intervention with a medium effect size for write-ups and a large effect size for restrictive housing. Despite similar rates of improvement for both groups, there were no statistically significant differences between the START NOW + BLS group and control group pre-intervention. Therefore, it appears that there is some sort of intervention occurring in the correctional facility that the 
control group is experiencing, as they are improving at a similar rate to those in the START NOW + BLS group.

There are limitations to the current study, including the potential constraint in the length of time chosen for the pre-intervention and post-intervention evaluation periods. Given some of the infrequency of occurrence in the outcome variables, it is possible that the three-month postintervention period was not long enough to yield significant differences between the groups. This facility is also structured in such a way that the SCTC and restrictive housing units share the same correctional staff (i.e., correctional officers, lieutenant). Thus, it is possible that due to staff exposure to the SCTC and this unit's rehabilitative approach to behavioral disruption, correctional staff have generalized this approach to the restrictive housing unit as well.

Additionally, in the current study, improvement in the control group may have been due to receiving other types of treatment (e.g., drug treatment, individual psychotherapy, sex offender programming) between the pre-intervention and post-intervention periods of observation. A portion of participants in the control group were convicted of a sex offense. Not all sex offenders are required to engage in sex offender programming; however, sex offenders who score in the top $90 \%$ for risk of recidivism, based on the Static-99R (i.e., assessing the level of risk for sex offense recidivism; Phenix, Fernandez, Harris, Helmus, Hanson, \& Thornton, 2016), are required to engage in 52 sessions of sex offender treatment. This treatment focuses on sex offense thinking errors, impulse control techniques, healthy relationships, and relapse prevention, and therefore, may have improved behavioral dysregulation in the control group. While inmates could potentially generalize these skills to other areas of their lives, this treatment occurs during their last two years incarcerated, prior to their parole or discharge date. Of the 43 participants in the control group, only 10 were close enough to parole or discharge to potentially 
meet this criterion. Of those 10 participants, only 1 had a sex offense charge. Therefore, it is unlikely that sex offender programming would have impacted the control group enough to account for the lack of significant data between the START NOW + BLS and control groups.

Despite methodological improvements, there were limitations to the current study, which may have contributed to the significant differences between the findings in this study and the previous START NOW intervention literature. Although the sample size was large enough for adequate power, it was not large enough to ensure adequate power to split groups (i.e., START NOW + BLS versus control) into subgroups (e.g., by mental health diagnoses). With a larger sample size, we may have been able to further group individuals and determine more definitively if these groupings (e.g., mental health diagnoses) impacted the effectiveness of the intervention. A majority of the outcome variables in the current study either had a low frequency of events (i.e., mental health referrals, precaution status, and use of force) or minimal variability between participants (i.e., restrictive housing). This was much lower than anticipated, as the inmates chosen for the SCTC are considered to be largely violent, aggressive, and disruptive. It is possible that the lack of frequency and variability of these events impacted the findings, leading to nonsignificant data. It is also possible that the low frequency and variability of these events was due to the chosen time frame (i.e., three months), and that with a lengthier evaluation period, there may be a higher frequency of these events. This appears to be especially relevant for the restrictive housing outcome variable, as inmates can receive administrative segregation (i.e., restrictive housing that is approved for a longer period of time). Administrative segregation would likely limit the variability of the data, as it typically lasts longer than three months (i.e., the length of the pre-intervention and post-intervention periods). However, researchers were not able to access these data to determine which inmates may have been placed on administrative 
segregation during the pre-intervention or post-intervention periods. Overall, the restrictive housing variable has a fixed time frame, as inmates in the DCR are typically sanctioned to 30 or 60 days based on their offense. Therefore, variability would continue to be limited, regardless of the time frame chosen for the pre-intervention and post-intervention period. Although it is possible that choosing a different time frame may have led to more variability, the chosen threemonth pre-intervention and post-intervention period is consistent with previous START NOW intervention research (Shelton \& Wakai, 2011), and was chosen in consultation with staff on-site at the correctional facility.

Additionally, due to feasibility, we were unable to obtain information about mental health treatment of the control group. It is possible that some inmates in the control group (although never having received the START NOW intervention or been placed in the SCTC) were engaging in individual psychotherapy, sex offender treatment, or group therapy related to substance use while inmates housed in the SCTC were completing the START NOW intervention, as these programs are also offered at this facility. These interventions could have improved their behavioral dysregulation from pre-intervention to post-intervention, although it is unlikely that a large portion of the control group all received these services during this particular time frame.

\section{Future Directions}

To improve future studies evaluating the START NOW intervention the following future directions are proposed. A portion of the measures included in the current study (i.e., number of mental health referrals, instances of use of force, and placement on precaution status) were limited in frequency of events. Additionally, it is unclear if these variables captured the behavioral dysregulation staff described of this inmate population. The inmates placed in the 
SCTC are described by correctional staff as largely aggressive, violent, and disruptive; however, through the use of these measures (i.e., number of mental health referrals, instances of use of force, and placement on precaution status), many inmates did not appear to have significant behavioral difficulties. Therefore, the addition of new variables to estimate behavior concerns, or different measurements of similar variables could lead to improvements in the frequency of the data, as well as provide a more accurate portrayal of the behavioral dysregulation the inmates in this program are described to have.

Additionally, there were a number of inmates in the SCTC since its inception who did not meet the inclusion criteria of completing at least 17 START NOW sessions for the current study. It is possible that these particular inmates exhibited such significant behavior difficulties (e.g., physical altercations with staff or inmates), that led to their removal from the SCTC. There are multiple different comparison groups that could be added to strengthen the methodology of the START NOW evidence base, as well as clarify some of the discrepancies between the current study and previous START NOW literature. Adding an additional comparison group comprised of data from inmates who were in the SCTC, but did not complete at least 17 START NOW sessions in future studies may provide further detail regarding the success of the program.

Although the control group in the current study appeared to be strong (i.e., no significant differences on pre-intervention outcome variables), a second possible control group, a waitlist control, may further strengthen the START NOW research base. It is unlikely that a majority of the inmates in the control group were engaging in other forms of treatment (e.g., sex offender treatment, substance use treatment). However, the inclusion of a third group of inmates who are not engaging in any form of treatment would further confirm these findings. 
Due to the discrepancy in results from the current study and the previous START NOW research, further research focused on replication is necessary. It is possible that this intervention does not improve behavioral difficulties further than living in the general population and receiving other, already existing, programs (e.g., sex offender programming, drug treatment, individual psychotherapy). However, this claim cannot be made without further research and replication.

Finally, obtaining a larger sample size that will allow for adequate power to split groups (i.e., START NOW + BLS versus control) into subgroups (e.g., mental health diagnoses) will strengthen the evidence base. This would determine if participants fit into subgroups (e.g., type of offense, time incarcerated), which then impact the effectiveness of the intervention.

\section{Conclusion}

Overall, the current study stands in contrast to previous START NOW intervention studies, as there were not meaningful differences between the START NOW + BLS group and the control group on any of the outcome variables. The START NOW intervention + BLS, did exhibit some success in reducing the number of write-ups and number of placements in restrictive housing; however, the control group also displayed success on these same variables. Although various limitations were identified, it is unlikely that those design changes would have impacted the results of the current study, as the changes would not have altered the behavioral improvement made by the control group. In summary, although the current study did not find that the START NOW plus behavioral level system group was significantly different from the control group on outcome variables, the intervention group did improve overall. Therefore, further research is necessary to determine the impact of this intervention on inmate disruptive, aggressive, and violent behavior. 


\section{References}

American Civil Liberties Union (2014). The dangerous overuse of solitary confinement in the United States. ACLU Foundation, 1-21. doi: 10.1163/2210-7975_hrd-9970-2014003

Atthowe, J. M., Jr., \& Krasner, L. (1968). Preliminary report on the application of contingent reinforcement procedures (token economy) on a "chronic" psychiatric ward. Journal of Abnormal Psychology, 73(1), 37-43. doi:10.1037/h0025439

Beck, A. J. (2015). Use of Restrictive Housing in U.S. Prisons and Jails, 2011-12. Bureau of Justice Statistics, 1-23.

Bonta, J., \& Andrews, D. A. (2007). Risk-need-responsivity model for offender assessment and rehabilitation. Rehabilitation, 6(1), 1-22.

Briggs, C. S., Sundt, J. L., \& Castellano, T. C. (2003). The effect of supermaximum security prisons on aggregate levels of institutional violence. Criminology, 41(4), 1341-1376. doi:10.1111/j.1745-9125.2003.tb01022.x

Broden, M., Hall, R. V., Dunlap, A., \& Clark, R. (1970). Effects of teacher attention and a token reinforcement system in a junior high school special education class. Exceptional Children, 36(5), 341-349. doi:10.1177/001440297003600505

Bronson, J., \& Berzofsky, M. (2017). Indicators of mental health problems reported by prisoners and jail inmates, 2011-12. Bureau of Justice Statistics, 1-16.

Browne, A., Hastings, A., Kall, K., \& diZerega, M. (2015). Keeping vulnerable populations safe under PREA: Alternative strategies to the use of segregation in prisons and jails. Vera Institute of Justice, 1-22.

Burdon, W. M., St. De Lore, J., Dang, J., Warda, U. S., \& Prendergast, M. L. (2013).

Psychosocial functioning among inmates in prison-based drug treatment: Results from 
Project BRITE. Journal of Experimental Criminology, 9(1), 45-64. doi:10.1007/s11292$012-9169-5$

Burdon, W. M., St. De Lore, J., \& Prendergast, M. L. (2011). Developing and implementing a positive behavioral reinforcement intervention in prison-based drug treatment: Project BRITE. Journal of Psychoactive Drugs, 43(1), 40-50. doi:10.1080/02791072.2011.601990

Cislo, A. M., \& Trestman, R. L. (2016). Psychiatric hospitalization after participation in START NOW. Psychiatric Services, 67(1), 143. doi:10.1176/appi.ps.201500456

Clark, V. A. (2015). Making the most of second chances: An evaluation of Minnesota's high-risk revocation reduction reentry program. Journal of Experimental Criminology, 11(2), 193215. doi: $10.1007 / \mathrm{s} 11292-014-9216-5$

Daffern, M., \& Howells, K. (2009). Self-harm and aggression in dangerous and severely personality disordered patients of a high-security hospital. Psychiatry, Psychology and Law, 16(1), 150-154. doi:10.1080/13218710802471156

Dixon, D. N., Heppner, P. P., Petersen, C. H., \& Ronning, R. R. (1979). Problem-solving workshop training. Journal of Counseling Psychology, 26(2), 133-139. doi:10.1037/0022-0167.26.2.133

Eccleston, L., \& Sorbello, L. (2002). The RUSH program-real understanding of self-help: A suicide and self-harm prevention initiative within a prison setting. Australian Psychologist, 37(3), 237-244. doi:10.1080/00050060210001706926

Frey, R. E. C., \& Weller, J. (2000). Rehab rounds: Behavioral management of aggression through teaching interpersonal skills. Psychiatric Services, 51(5), 607-609. doi:10.1176/appi.ps.51.5.607 
Garofalo, C., Velotti, P., \& Zavattini, G. C. (2018). Emotion regulation and aggression: The incremental contribution of alexithymia, impulsivity, and emotion dysregulation facets. Psychology of Violence, 8(4), 470-483. doi:10.1037/vio0000141

Gendreau, P. (1996). Offender rehabilitation: What we know and what needs to be done. Criminal Justice and Behavior, 23(1), 144-161. doi:10.1177/0093854896023001010

Glowacki, K., Warner, G., \& White, C. (2016). The use of a token economy for behaviour and symptom management in adult psychiatric inpatients: A critical review of the literature. Journal of Psychiatric Intensive Care, 12(2), 119-127. doi:10.20299/jpi.2016.009

Glowa-Kollisch, S., Kaba, F., Waters, A., Leung, Y., Ford, E., \& Venters, H. (2016). From punishment to treatment: The "clinical alternative to punitive segregation" (CAPS) program in New York City jails. International Journal of Environmental Research and Public Health, 13(2), 182-191. doi:10.3390/ijerph13020182

Gordon, J. A. (1999). Do staff attitudes vary by position?: A look at one juvenile correctional center. American Journal of Criminal Justice, 24(1), 81-93. doi:10.1007/bf02887619

Hayakawa, M. (2009). How repeated 15-minute assertiveness training sessions reduce wrist cutting in patients with borderline personality disorder. American Journal of Psychotherapy, 63(1), 41-51. doi:10.1176/appi.psychotherapy.2009.63.1.41

Hesser, H., Axelsson, S., Bäcke, V., Engstrand, J., Gustafsson, T., Holmgren, E., ... Andersson, G. (2017). Preventing intimate partner violence via the internet: A randomized controlled trial of emotion-regulation and conflict-management training for individuals with aggression problems. Clinical Psychology \& Psychotherapy, 24(5), 1163-1177. doi:10.1002/cpp.2082 
Kaba, F., Lewis, A., Glowa-Kollisch, S., Hadler, J., Lee, D., Alper, H., ... Venters, H. (2014). Solitary confinement and risk of self-harm among jail inmates. American Journal of Public Health, 104(3), 442-447. doi:10.2105/ajph.2013.301742

Kaeble, D., \& Cowhig, M. (2018). Correctional Populations in the United States, 2016. Bureau of Justice Statistics, 1-13.

Kersten, L., Cislo, A. M., Lynch, M., Shea, K., \& Trestman, R. L. (2015). Evaluating START NOW: A skills-based psychotherapy for inmates of correctional systems. Psychiatric Services, 67(1), 37-42. doi:10.1176/appi.ps.201400471

Koons, C. R., Chapman, A. L., Betts, B. B., O’Rourke, B., Morse, N., \& Robins, C. J. (2006). Dialectical behavior therapy adapted for the vocational rehabilitation of significantly disabled mentally ill adults. Cognitive and Behavioral Practice, 13(2), 146-156. doi:10.1016/j.cbpra.2005.04.003

Kurtz, P. F., Chin, M. D., Huete, J. M., Tarbox, R. S. F., O’Connor, J. T., Paclawskyj, T. R., \& Rush, K. S. (2003). Functional analysis and treatment of self-injurious behavior in young children: A summary of 30 cases. Journal of Applied Behavior Analysis, 36(2), 205-219. doi:10.1901/jaba.2003.36-205

Lanes, E. (2009). The association of administrative segregation placement and other risk factors with the self-injury-free time of male prisoners. Journal of Offender Rehabilitation, 48(6), 529-546. doi:10.1080/10509670903081342

Linehan, M. M. (2015). DBT Skills Training Manual (2nd ed.). New York, NY: The Guilford Press. 
Linehan, M. M., Armstrong, H. E., Suarez, A., Allmon, D., \& Heard, H. L. (1991). Cognitivebehavioral treatment of chronically parasuicidal borderline patients. Archives of General Psychiatry, 48(12), 1060-1064. doi:10.1001/archpsyc.1991.01810360024003

Linehan, M. M., Tutek, D. A., Heard, H. L., \& Armstrong, H. E. (1994). Interpersonal outcome of cognitive behavior treatment for chronically suicidal borderline patients. American Journal of Psychiatry, 151(12), 1771-1775. doi:10.1176/ajp.151.12.1771

Low, G., Jones, D., Duggan, C., MacLeod, A., \& Power, M. (2001). Dialectical behaviour therapy as a treatment for deliberate self-harm: Case studies from a high security psychiatric hospital population. Clinical Psychology \& Psychotherapy, 8(4), 288-300. doi:10.1002/cpp.287

McCredie, M. N., Quinn, C. A., \& Covington, M. (2017). Dialectical behavior therapy in adolescent residential treatment: Outcomes and effectiveness. Residential Treatment for Children and Youth, 34(2), 84-106. doi:10.1080/0886571x.2016.1271291

Mennin, D. S., Fresco, D. M., O’Toole, M. S., \& Heimberg, R. G. (2018). A randomized controlled trial of emotion regulation therapy for generalized anxiety disorder with and without co-occurring depression. Journal of Consulting and Clinical Psychology, 86(3), 268-281. doi:10.1037/ccp0000289

Miller, H. A., \& Young, G. R. (1997). Prison segregation: Administrative detention remedy or mental health problem? Criminal Behaviour and Mental Health, 7(1), 85-94. doi:10.1002/cbm.146

Morris, R. G. (2016). Exploring the effect of exposure to short-term solitary confinement among violent prison inmates. Journal of Quantitative Criminology, 32(1), 1-22. doi:10.1007/s10940-015-9250-0 
National Commission on Correctional Healthcare (2016). Position statement: Solitary confinement (isolation). 1-6.

National Commission on Correctional Healthcare (2018). Suicide prevention and intervention. Standards for health services in prisons (pp. 39-41).

O’Donnell, O., House, A., \& Waterman, M. (2015). The co-occurrence of aggression and selfharm: Systematic literature review. Journal of Affective Disorders, 175, 325350. doi:10.1016/j.jad.2014.12.051

Oklahoma Department of Corrections (2018). Use of force standards and reportable incidents, 126.

Phenix, A., Fernandez, Y., Harris, A. J. R., Helmus, M., Hanson, R. K., \& Thornton, D. (2016). Static-99R Coding Rules Revised-2016 (pp. 1-94). Available at www.static99.org Pistorello, J., Fruzzetti, A. E., MacLane, C., Gallop, R., \& Iverson, K. M. (2012). Dialectical behavioral therapy (DBT) applied to college students: A randomized clinical trial. Journal of Consulting and Clinical Psychology, 80(6), 982-994. doi:10.1037/a0029096

Pritchard, D., Penney, H., \& Mace, F. C. (2017). The ACHIEVE! program: A point and level system for reducing severe problem behavior. Behavioral Interventions, 33(1), 41-55. doi:10.1002/bin. 1506

Rollnick, S., \& Miller, W. R. (1995). What is motivational interviewing? Behavioural and Cognitive Psychotherapy, 23(4), 325-334. doi:10.1017/s135246580001643x

Roush, A., Bryson, J., \& Weaver, J. (2012). Introduction to the Behavior Health Unit. State of West Virginia Division of Corrections, 1-8.

Sampl, S., Trestman, R. L., \& Krauss, W. J. (2013). START NOW Facilitator Manual. Farmington, CT: University of Connecticut Health Center. 
Sampl, S., Wakai, S., \& Trestman, R. L. (2010). Translating evidence-based practices from community to corrections: An example of implementing DBT-CM. The Journal of Behavior Analysis of Offender and Victim Treatment and Prevention, 2(2), 114-123. doi:10.1037/h0100463

Sampl, S., Wakai, S., Trestman, R. L., \& Keeney, E. M. (2008). Functional analysis of behavior in corrections: Empowering inmates in skills training groups. The Journal of Behavior Analysis of Offender and Victim Treatment and Prevention, 1(4), 42-51. doi:10.1037/h0100455

Shelton, D., Kesten, K., Zhang, W., \& Trestman, R. (2011). Impact of a dialectic behavior therapy-corrections modified (DBT-CM) upon behaviorally challenged incarcerated male adolescents. Journal of Child and Adolescent Psychiatric Nursing, 24(2), 105-113. doi:10.1111/j.1744-6171.2011.00275.x

Shelton, D., Sampl, S., Kesten, K. L., Zhang, W., \& Trestman, R. L. (2009). Treatment of impulsive aggression in correctional settings. Behavioral Sciences and the Law, 27(5), 787-800. doi:10.1002/bsl.889

Shelton, D., \& Wakai, S. (2011). A process evaluation of START NOW skills training for inmates with impulsive and aggressive behaviors. Journal of the American Psychiatric Nurses Association, 17(2), 148-157. doi:10.1177/1078390311401023

Shayne, R., \& Miltenberger, R. G. (2013). Evaluation of behavioral skills training for teaching functional assessment and treatment selection skills to parents. Behavioral Interventions, 28(1), 4-21. doi:10.1002/bin.1350 
Suedfeld, P., Ramirez, C., Deaton, J., \& Baker-Brown, G. (1982). Reactions and attributes of prisoners in solitary confinement. Criminal Justice and Behavior, 9(3), 303-340. doi:10.1177/0093854882009003004

Tartaro, C. (2015). An evaluation of the effects of jail diversion and reentry for mentally ill offenders. Journal of Offender Rehabilitation, 54(2), 85-102. doi:10.1080/10509674.2015.1009966

The Sentencing Project (2017). The facts: State-by-state data. The Sentencing Project.

Tomlinson, M. F. (2018). A theoretical and empirical review of dialectical behavior therapy within forensic psychiatric and correctional settings worldwide. International Journal of Forensic Mental Health, 17(1), 72-95. doi:10.1080/14999013.2017.1416003

Travis, J., Western, B., \& Redburn, F. S. (2014). The growth of incarceration in the United States: Exploring causes and consequences. Washington D.C.: The National Academies Press. doi:10.17226/18613

United States Government Accountability Office (2013). Improvements Needed in Bureau of Prisons’ Monitoring and Evaluation of Impact of Segregated Housing. 1-66.

Van Allen, R. K. (1970). Improvement in appearance and grooming in a token-economy setting as a function of reinforcement contingency. Canadian Journal of Behavioural Science / Revue Canadienne Des Sciences Du Comportement, 2(2), 157-161. doi:10.1037/h0082719

van den Bosch, L., Verheul, R., Schippers, G. M., \& van den Brink, W. (2002). Dialectical behavior therapy of borderline patients with and without substance use problems: Implementation and long-term effects. Addictive Behaviors, 27(6), 911-923. doi:10.1016/s0306-4603(02)00293-9 
Verheul, R., van den Bosch, L. M., Koeter, M. W., de Ridder, M. A., Seijnen, T., \& van den Brink, W. (2003). Dialectical behavior therapy for women with borderline personality disorder: 12-month, randomised clinical trial in The Netherlands. British Journal of Psychiatry, 182(2), 135-140. doi:10.1192/bjp.182.2.135

Welches, P., \& Pica, M. (2005). Functional analysis of behavior: A collaborativephenomenological approach. The Humanistic Psychologist, 33(1), 59-68. doi:10.1207/s15473333thp3301_6

West Virginia Division of Corrections and Rehabilitation (2013). Discipline of inmates, 1-39. Wetterborg, D., Dehlbom, P., Långström, N., Andersson, G., Fruzzetti, A. E., \& Enebrink, P. (2020). Dialectical behavior therapy for men with borderline personality disorder and antisocial behavior: A clinical trial. Journal of Personality Disorders, 34(1), 22-39. doi:10.1521/pedi_2018_32_379

Zinger, I., Wichmann, C., \& Andrews, D. A. (2001). The psychological effects of 60 days in administrative segregation. Canadian Journal of Criminology, 43(1), 47-83. doi:10.22215/etd/1999-04119 
Table 1

\begin{tabular}{|c|c|c|c|c|c|c|c|c|c|c|c|}
\hline \multicolumn{12}{|c|}{ Means and Standard Deviations for Conditions } \\
\hline & & $\begin{array}{c}\text { Pre- } \\
\text { Intervention } \\
\text { Mental } \\
\text { Health } \\
\text { Referrals }\end{array}$ & $\begin{array}{c}\text { Post- } \\
\text { Intervention } \\
\text { Mental } \\
\text { Health } \\
\text { Referrals }\end{array}$ & $\begin{array}{c}\text { Pre- } \\
\text { Intervention } \\
\text { Use of } \\
\text { Force }\end{array}$ & $\begin{array}{c}\text { Post- } \\
\text { Intervention } \\
\text { Use of } \\
\text { Force }\end{array}$ & $\begin{array}{c}\text { Pre- } \\
\text { Intervention } \\
\text { Restrictive } \\
\text { Housing }\end{array}$ & $\begin{array}{c}\text { Post- } \\
\text { Intervention } \\
\text { Restrictive } \\
\text { Housing }\end{array}$ & $\begin{array}{c}\text { Pre- } \\
\text { Intervention } \\
\text { Write-ups }\end{array}$ & $\begin{array}{c}\text { Post- } \\
\text { Intervention } \\
\text { Write-ups }\end{array}$ & $\begin{array}{c}\text { Pre- } \\
\text { Intervention } \\
\text { Precaution } \\
\text { Status }\end{array}$ & $\begin{array}{c}\text { Post- } \\
\text { Intervention } \\
\text { Precaution } \\
\text { Status }\end{array}$ \\
\hline \multirow[t]{2}{*}{$\begin{array}{l}\text { START } \\
\text { NOW + } \\
\text { BLS }\end{array}$} & $\begin{array}{c}M \\
(S D)\end{array}$ & $\begin{array}{c}.16 \\
(.55)\end{array}$ & $\begin{array}{c}.23 \\
(.97)\end{array}$ & $\begin{array}{c}.44 \\
(1.01)\end{array}$ & $\begin{array}{c}.36 \\
(1.13)\end{array}$ & $\begin{array}{l}1.20 \\
(.66)\end{array}$ & $\begin{array}{c}.58 \\
(.72)\end{array}$ & $\begin{array}{c}1.57 \\
(1.86)\end{array}$ & $\begin{array}{c}1.14 \\
(1.82)\end{array}$ & $\begin{array}{c}.24 \\
(.65)\end{array}$ & $\begin{array}{c}.18 \\
(.49)\end{array}$ \\
\hline & $N$ & 38 & 40 & 45 & 45 & 45 & 45 & 42 & 42 & 45 & 45 \\
\hline \multirow[t]{2}{*}{ Control } & $\begin{array}{c}M \\
(S D)\end{array}$ & $\begin{array}{c}.03 \\
(.16)\end{array}$ & $\begin{array}{c}.03 \\
(.16)\end{array}$ & $\begin{array}{c}.26 \\
(.62)\end{array}$ & $\begin{array}{c}.16 \\
(.65)\end{array}$ & $\begin{array}{l}1.14 \\
(.41)\end{array}$ & $\begin{array}{c}.58 \\
(.76)\end{array}$ & $\begin{array}{c}.80 \\
(1.22)\end{array}$ & $\begin{array}{c}.48 \\
(.91)\end{array}$ & $\begin{array}{c}.07 \\
(.46)\end{array}$ & $\begin{array}{c}.07 \\
(.34)\end{array}$ \\
\hline & $N$ & 40 & 40 & 43 & 43 & 43 & 43 & 40 & 40 & 43 & 43 \\
\hline
\end{tabular}

Note. Variations in participant number are due to missing data in inmate records or removal of outliers. 
Table 2.

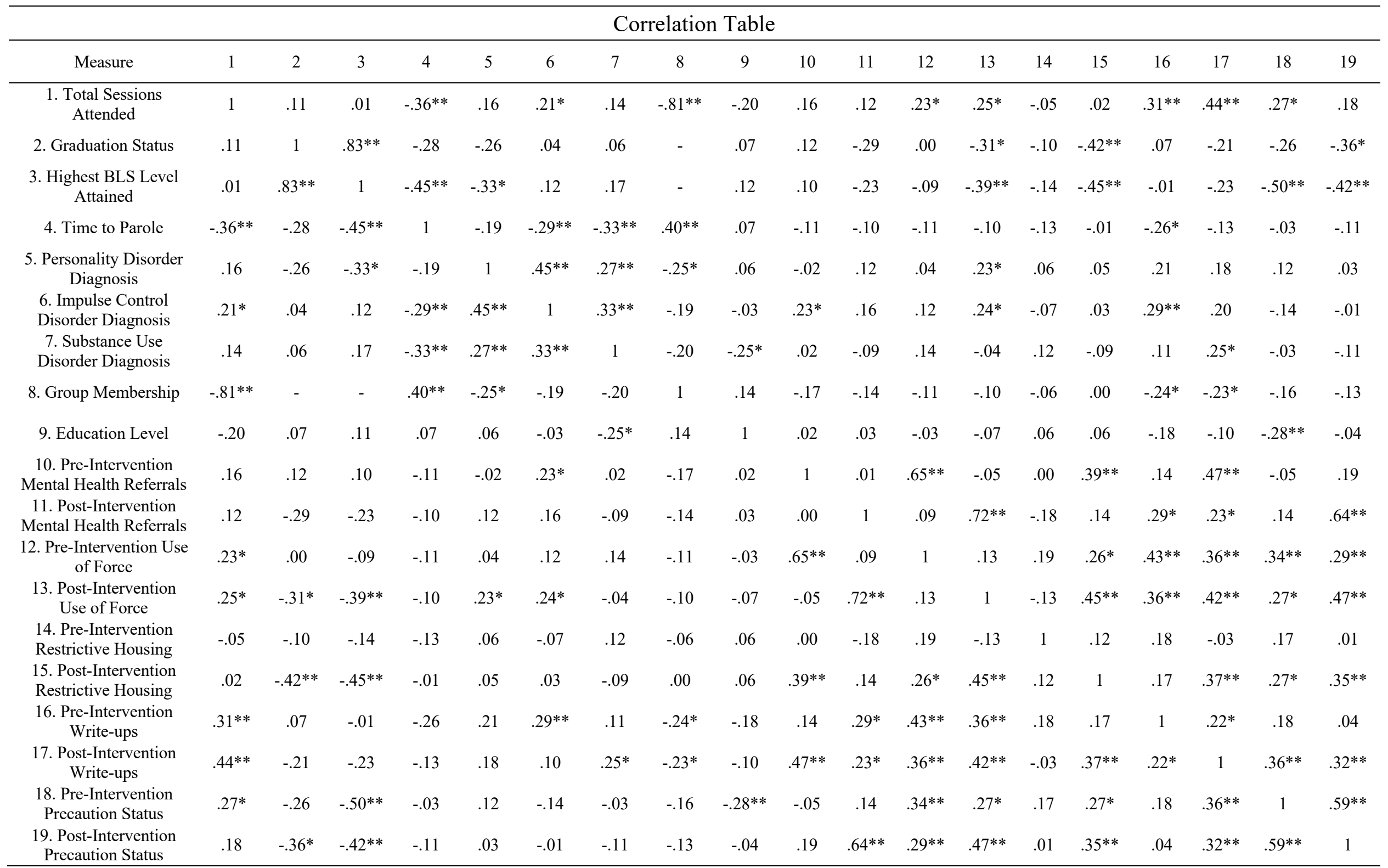

Note. ${ }^{* *} \mathrm{p}<.01,{ }^{*} \mathrm{p}<.05$ 